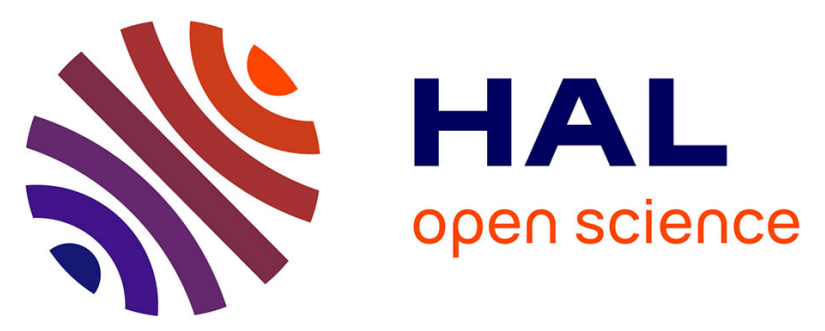

\title{
The chemical continuous time random walk framework for upscaling transport limitations in fluid-solid reactions
}

Tomás Aquino, Tanguy Le Borgne

\section{- To cite this version:}

Tomás Aquino, Tanguy Le Borgne. The chemical continuous time random walk framework for upscaling transport limitations in fluid-solid reactions. Advances in Water Resources, In press, pp.103981. 10.1016/j.advwatres.2021.103981 . insu-03261754v1

\section{HAL Id: insu-03261754 \\ https://hal-insu.archives-ouvertes.fr/insu-03261754v1}

Submitted on 16 Jun 2021 (v1), last revised 22 Jun 2021 (v2)

HAL is a multi-disciplinary open access archive for the deposit and dissemination of scientific research documents, whether they are published or not. The documents may come from teaching and research institutions in France or abroad, or from public or private research centers.
L'archive ouverte pluridisciplinaire HAL, est destinée au dépôt et à la diffusion de documents scientifiques de niveau recherche, publiés ou non, émanant des établissements d'enseignement et de recherche français ou étrangers, des laboratoires publics ou privés. 


\section{Journal Pre-proof}

The chemical continuous time random walk framework for upscaling transport limitations in fluid-solid reactions

Tomás Aquino, Tanguy Le Borgne

PII: S0309-1708(21)00136-6

DOI: https://doi.org/10.1016/j.advwatres.2021.103981

Reference: ADWR 103981

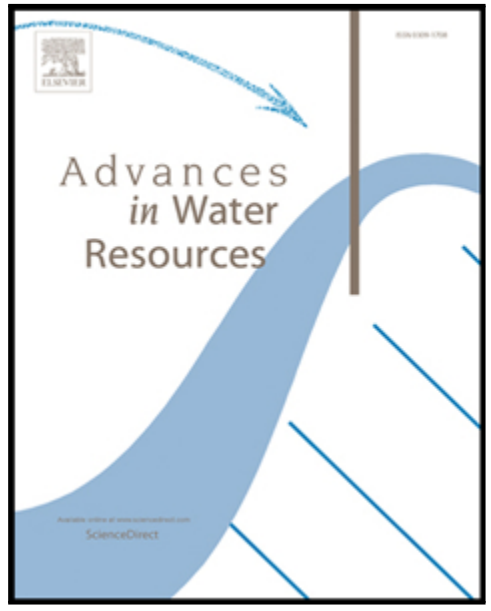

To appear in:

Advances in Water Resources

Received date:

19 April 2021

Revised date:

4 June 2021

Accepted date:

8 June 2021

Please cite this article as: Tomás Aquino, Tanguy Le Borgne, The chemical continuous time random walk framework for upscaling transport limitations in fluid-solid reactions, Advances in Water Resources (2021), doi: https://doi.org/10.1016/j.advwatres.2021.103981

This is a PDF file of an article that has undergone enhancements after acceptance, such as the addition of a cover page and metadata, and formatting for readability, but it is not yet the definitive version of record. This version will undergo additional copyediting, typesetting and review before it is published in its final form, but we are providing this version to give early visibility of the article. Please note that, during the production process, errors may be discovered which could affect the content, and all legal disclaimers that apply to the journal pertain.

(C) 2021 Published by Elsevier Ltd. 


\section{Highlights}

- We introduce the concept of inter-reaction times for quantifying transport limitations in fluid-solid reactions

- We upscale this process using the chemical continuous time random walk framework

- Results are compared to two- and three-dimensional particle tracking simulations 
Fluid-solid reactions play a key role in a large range of biogeochemical processes. Transport limitations at the pore scale limit the amount of solute available for reaction, so that reaction rates measured under well-mixed conditions tend to strongly overestimate rates occurring in natural and engineered systems. Although different models have been proposed to capture this phenomenon, linking pore-scale structure, flow heterogeneity, and local reaction kinetics to upscaled effective kinetics remains a challenging problem. We present a new theoretical framework to quantify these dynamics based on the chemical continuous time random walk framework. We study a fluidsolid reaction with the fluid phase undergoing advective-diffusive transport. We consider a catalytic degradation reaction, $A_{F}+B_{S} \rightarrow B_{S}$, where $A_{F}$ is in fluid phase and $B_{S}$ is in solid phase and homogeneous over the fluidsolid interface, allowing us to focus on the role of transport limitations and medium structure. Our approach is based on the concept of inter-reaction times, which result from the times between contacts of transported reactants with the solid phase. We use this formulation to quantify the global kinetics of fluid-reactant mass and test our predictions against numerical simulations of advective-diffusive transport in stratified channel flow and Stokes flow through a beadpack. The theory captures the decrease of effective reaction rates compared to the well-mixed prediction with increasing Damköhler number due to transport limitations. Although we consider simple kinetics and media, these findings will contribute to the understanding and modeling of the effect of transport limitations in more complex reactive transport problems.

14

Keywords: Reactive transport, Stochastic modeling, Chemical continuous

Prepkints sybmitted tothduances in Water Resources

Email address: tomas.decamposaquino@univ-rennes1.fr (Tomás Aquino) 
time random walk

\section{Introduction}

Biogeochemical reactions at the interface between fluid and solid medium phases play a central role in a large range of reactive transport problems, such as contaminant transport and degradation, soil remediation, mineral weathering, and carbon dioxide sequestration [5-7]. Biotic and abiotic reactions at solid-fluid interfaces include dissolution/precipitation, adsorption, complexation and redox reactions. The kinetics of these reactions on solid surfaces depend directly on the concentration of solutes in the fluid phase, which evolve in time and space through flow and transport dynamics. Therefore, much effort has been invested into the development, setup, and choice of detailed reactive transport models to quantify these processes and their interaction with transport and medium geometry [1-3]. The basic quantification of the kinetics of such reactions is generally performed using wellmixed batch experiments. Yet, transport limitations at the pore scale lead to large deviations from these estimates by reducing access of solutes to reactive surfaces compared to fully-mixed systems. This phenomenon has been observed in resolved numerical simulations of carbonate mineral dissolution in porous media [8], numerical simulation and column experiments of calcite dissolution $[9,21,22]$, numerical simulations of mineral dissolution in heterogeneous porous media $[11,23]$, pore-scale reactive transport simulations in rough fractures [13], and batch experiments and field-scale modeling of biodegradation of dissolved organic carbon in aquifers [24]. Pore-scale flow and structure have also been found to significantly impact adsorption to mineral surfaces in porous media, an effect which has been observed in detailed lattice-Boltzmann simulations [16-18].

These studies have consistently found that reaction rates are significantly lower than expected from classical well-mixed theories, especially when reaction is fast compared to transport processes. Volume averaging techniques $[14,15]$ have been employed to identify general conditions under which classical macroscopic models of reactive transport break down, and transport limitations lead to decreased global reaction rates and/or modified rate laws. Random walk models have been used to investigate the impact of transport on surface reactions for simple geometries, such as sinusoidal channels $[19,20]$. The role of available reactive surface area $[10,12]$ and surface roughness [13] in mineral dissolution in porous and fractured media 
has been analyzed and quantified through experiments and numerical simulations. However, a quantitative link between pore-scale transport dynamics and effective fluid-solid reaction kinetics remains unavailable. Furthermore, in practice, highly-resolved numerical simulations can be prohibitively expensive, and detailed knowledge of the dynamics and spatial distribution of physico-chemical properties is often not available, stressing the need for upscaled models of reactive transport [26].

In well-mixed batch reactors, reactant concentrations are spatially homogeneous. In the Lagrangian particle picture, this corresponds to every particle being instantaneously available to participate in a reaction with every other particle. This deterministic picture can be extended to account for stochastic variability for small particle numbers, while retaining the wellmixed assumption [28]. This is achieved through the concept of inter-reaction times, which represent the time between the occurrence of sequential reaction events amongst sets of reactants in accordance with the chemical reactions. In the classical stochastic theory, reactants are assumed to be fully-mixed, in the sense that all sets of reactants allowed by the chemistry have the same probability of reacting. This leads to exponentially-distributed interreaction times, representing a probability per unit time of reaction that is fully determined by the thermodynamic reaction rate and the available reactant numbers at a given time. The classical well-mixed rate laws, under which reaction rates correspond to products of reactant concentrations with powers determined by the reaction stoichiometry, are recovered in the limit of large particle numbers [29]. In practice, this picture holds only if diffusion is sufficiently fast to locally homogenize reactants, so that the limiting factor in determining reaction rates lies in the thermodynamic properties of the reaction rather than transport.

Fluid-solid reactions involve transported and immobile reactants. Solidphase reactants are located at the interface between a fluid phase, in which fluid-phase reactants are transported, and a solid phase of the underlying medium. The first explicit model of the impact of transport on reaction is due to von Smoluchowski [30]. It quantifies contact reactions between a hard sphere and a sea of diffusing particles, and it leads to an effective, time-dependent reaction rate which depends on transport properties, namely the diffusion coefficient. Because there is no fluid flow into or out of the solid interface, mass flux of fluid reactants allowing contact with solid-phase reactants is ultimately governed by diffusion. On the other hand, advective transport along streamlines may bring reactants closer or farther from the 
solid phase. Thus, in the inter-reaction time picture discussed above, the combined effect of medium heterogeneity, advective variability, and diffusion introduces reaction delays in terms of the first passage times of reactants to the solid phase. Quantifying this effect and its impact on reaction rates is therefore fundamental for modeling fluid-solid reactions in porous and fractured media.

Recently, the chemical continuous time random walk (chCTRW) framework was developed in order to relax the well-mixed assumption in stochastic reaction modeling, leading to inter-reaction times which encode the effect of local transport limitations through additional reaction delays due to transport limitations [31]. The chCTRW hence quantifies the effect of broader distributions of the times required for sequential reaction events to occur. Despite the formal similarities, this differs conceptually from the classical CTRW framework, which quantifies the effect of broadly distributed times or distances associated with particle displacements [32]. Such reaction delays can be quantified in terms of the first passage times of reactant particles across each other [33-37]. In the case of fluid-solid reactions, these are related to the duration of excursions between visits to the solid interface. The latter are closely related to the time spent near the interface, which can be formally quantified through the so-called local time at the boundary, which represents the amount of time spent in a thin region near the interface divided by the region thickness, in the limit of vanishing thickness [38-41]. The concept of modeling reactive transport in terms of exposure time, that is, the time that reactants spend in close proximity and so are available for reaction, has received some attention over the past decade [42-46]. Nonetheless, the relationship between exposure time and flow and medium heterogeneity remains little understood. The central goal of the present work is to formalize the notion of inter-reaction times and their impact on reaction dynamics in the context of fluid-solid reactions under advective-diffusive transport, in order to better undertand and upscale the impact of flow, transport, and medium structure on global reaction rates.

We consider here a catalytic degradation reaction, $A_{F}+B_{S} \rightarrow B_{S}$, a simplified chemical setup which allows us to focus on the role of transport limitations. The reactant species $B_{S}$ is taken to be in solid phase, immobile, and homogeneously distributed over the fluid-solid interface, whereas the reactant species $A_{F}$ is in fluid phase and undergoes advective-diffusive transport. The impact of disordered (i.e., random and uncorrelated at different spatial locations) distributions of solid-phase reactants and residence times 
on this type of reaction has been studied for diffusive and subdiffusive transport (i.e., transport phenomena where plume variance grows sublinearly in time) and trapping using random walk models [48-55], and purely-advective transport in a streamtube model using the chCTRW framework [47]. However, these models did not consider the joint effect of flow variability and diffusion. In porous media, the interplay between these processes controls mass fluxes towards the fluid-solid interface, and therefore the amount of reactant available for reaction. As shown here, the interplay between medium geometry and transport limitations can lead to effective reaction kinetics that differ from their well-mixed counterparts, even for this simple chemical setup. It should be noted that we disregard for the present more complex effects which may play an important role in reactive transport dynamics, such as the coupling of transport and medium evolution due to reaction-induced precipitation and dissolution [56-60]. Our simplified setup allows for in-depth understanding and quantification of the specific role of transport limitations and medium geometry regarding global reaction dynamics, and provides a rigorous upscaling approach to be later extended to more complex reaction chemistry.

The paper is structured as follows. We first formalize fluid-solid reaction dynamics under diffusive transport near an interface in Section 2. This is followed by a brief review of the fundamental concepts behind inter-reaction times and the chCTRW formulation in Section 3. In Section 4, we develop the relationship between return times to the interface and inter-reaction times, and use this formulation to quantify the time evolution of total fluid-reactant mass. Next, in Section 5, we illustrate these results by obtaining an analytical formulation of the mass dynamics for advection-diffusion under stratified flow in a two-dimensional channel. Section 6 shows how the framework thus developed may be applied to compute the time evolution of total mass from numerical determination of first passage and return times in more general settings. In particular, we consider advection-diffusion under stratified flow in a three-dimensional channel and Stokes flow in an idealized porous medium, specifically a body centered cubic beadpack. An overall discussion and conclusions are presented in Section 7, and some additional technical details and derivations may be found in appendix. 


\section{Fluid--solid reaction model}

We consider a mobile reactant species $A_{F}$, transported by the fluid phase, and an immobile, solid-phase reactant species $B_{S}$, distributed over the fluidsolid interface of the medium. In order to focus on the effects of transport limitations, we assume for simplicity that the distribution of the latter over the interface is homogeneous, and that its concentration at a given spatial location does not change appreciably due to reaction. Assuming further that the reaction is irreversible at the timescale of interest and ignoring the reaction products, this corresponds locally to the reaction $A_{F}+B_{S} \rightarrow B_{S}$. We thus consider a far-from-equilibrium situation where the reverse reaction can be neglected. Mass conservation requires this reaction to give rise to additional products, which are ignored here. We consider also that the available reactant $B_{S}$ is homogeneous across the solid phase. While this assumption should not be expected to hold over large scales, it is directly relevant for relatively chemically-homogeneous column experiments, or over certain regions of larger media. The assumption that $\hat{B}_{S}$ is not consumed holds directly for truly catalytic reactions, but, along with homogeneity, it is also a relevant approximation if $B_{S}$ is locally not consumed appreciably, for example under large flow rates and short injections, where the fluid phase may be significantly consumed throughout the column, but consumption of the solid phase at a particular location is small. As mentioned in the introduction, the assumption of no consumption of the solid phase also implies that we also disregard more complex effects such as coupling of transport and medium evolution, which can occur due to precipitation and/or dissolution.

The resolved simulation method developed in this work can in principle handle more complex chemical setups, including multiple reactions and/or multicomponent reactions. However, the theoretical developments become substantially more complex, because it is necessary to account for the simultaneous presence and amount of different reactants near the interface. The simple chemical reaction $A_{F}+B_{S} \rightarrow B_{S}$, along with the assumption of chemical homogeneity of the solid-phase reactant, allows us to focus on the impact of transport mechanisms and medium geometry on reaction dynamics. Despite the fact that the chemical kinetics are linear at the fluid-solid interface, reaction is limited by the available fluid-reactant flux toward the latter, and transport limitations can lead to modified effective reaction kinetics and significant reaction slowdown. While it is important to note that the theory developed here cannot at present be directly applied to multicompo- 
nent chemical reactions, it provides the first direct link between first passage and return time statistics, inter-reaction times, and fluid-solid reaction dynamics, and sets the stage for later generalizations.

In order for reaction to occur, physical reactant molecules of the transported phase $A_{F}$ must be in reactive contact with the solid-phase component $B_{S}$. This occurs within some distance $\ell_{s}$ of the fluid-solid interface. We assume that $\ell_{s}$ is much smaller than the scale at which transport of reactant $A_{F}$ within the fluid phase may be described through continuous advectiondiffusion, and that reaction then occurs when reactant $A_{F}$ is transported sufficiently close to the interface. As we will see, this corresponds to the usual concept of surface (as opposed to bulk) reactions. It should be noted that if this spatial scale separation between transport and reaction does not hold, a more detailed reaction model is necessary. For example, if attachment or transport times within a physical reactive layer play a significant role, sorption or microporosity models may be needed, which we do not consider here.

From both a theoretical and a numerical perspective, it is convenient to adopt a conceptualization of transport in terms of Lagrangian tracer particles. Each Lagrangian particle represents a macroscopic number of physical reactant particles undergoing advection-diffusion [61, 62] and subject to reaction near the interface. Disregarding reaction for the moment, particle positions $\boldsymbol{X}(t)$ as a function of time $t$ are described by the Langevin equation (see, e.g., [63])

$$
d \boldsymbol{X}(t)=\boldsymbol{v}[\boldsymbol{X}(t)] d t+\sqrt{2 D d t} \boldsymbol{\xi}(t),
$$

where $D$ is the diffusion coefficient, $\boldsymbol{v}(\cdot)$ is the velocity field as a function of position, and, for each time $t, \boldsymbol{\xi}(t)$ are independent random vectors whose components are independent unit Gaussian random variables.

Under the assumed scale separation between reaction and transport, reaction dynamics are controlled by the concentration of fluid reactant $A_{F}$ near the interface, subject to both advective-diffusive transport and reaction. When a discretization is considered, for a given time step $\Delta t$, the support scale $\ell_{d}$ over which concentrations are well defined must be large compared to the spatial resolution of the model description, which is of order $\sqrt{2 D \Delta t}$ (see Appendix A for further details). It is important to note that $\ell_{d}$ is not a parameter of the theoretical model, but rather a property of the discretization. In this sense, the dynamics will be shown to converge to a 
well-defined limit when $\ell_{d} \rightarrow 0$, which corresponds to taking the continuum limit $\Delta t \rightarrow 0$.

For a given discretization, below the support scale $\ell_{d}$, the mass represented by a Lagrangian particle is taken to be well-mixed. Particles within a region comprising a distance up to $\ell_{d}$ from the interface are subject to reaction, as we will formalize below. In what follows, we will refer to this region as the reactive region for convenience, although it should be remembered that it is associated with a given discretization and different from the physical reactive region, associated with the subscale length $\ell_{s}$. In Section 4 , we will employ the chCTRW inter-reaction times to show that the continuum limit $\ell_{d} \rightarrow 0$ (or, equivalently, $\Delta t \rightarrow 0$ ) of this conceptualization is well defined and leads to a consistent description of the full reactive transport problem. This approach is conceptually convenient for the derivations that follow. From a computational standpoint, it converges to the correct results, as will be discussed in detail below. Nonetheless, we expect that it will prove useful in the future to explore equivalent but computationally more efficient approaches for direct numerical simulations, such as kernel-smoothing [64, 65] to determine concentrations near the interface. We note that any method for estimating local mass fluxes to the interface from Lagrangian particle collisions must address the same conceptual issues, since, as discussed in detail in what follows, the number of collisions with the interface within a given time period is also discretization-dependent.

According to the previous considerations, while a Lagrangian particle is in the reactive region, a fraction $\ell_{s} / \ell_{d}$ of its mass $M_{p}$ is physically available for reaction. Assuming the law of mass action holds locally, we have

$$
\frac{d M_{p}}{d t}=-k c_{s} \frac{\ell_{s} M_{p}}{\ell_{d}}
$$

where $k$ is the usual well-mixed reaction rate, in units of inverse concentration per time, and $c_{s}$ is the solid-phase concentration within the reactive region. Thus,

$$
\frac{d M_{p}}{d t}=-k_{d} M_{p}, \quad k_{d}=k \frac{c_{A}}{\ell_{d}},
$$

where $k_{d}$ is the effective particle reaction rate in units of inverse time, and $c_{A}=c_{s} \ell_{s}$ is the solid-phase surface concentration, i.e., mass of solid reactant per unit interface area. Note that, under the scale separation assumption, 
$\ell_{s}$ is small compared to the scale at which transport can be described by continuous advection-diffusion. This means that, formally, the continuum description then corresponds to the scaling limit $\ell_{d} \rightarrow 0$ with the physical concentration $c_{A}$ remaining finite, whereas $\ell_{s} / \ell_{d}$ remains small due to the scale separation. This scale separation corresponds to the situation where the fluid-solid mass-action reaction can be treated as a surface reaction: the reaction rate $k_{d}$, describing particle mass decay per unit time in the reactive region, is independent of $\ell_{s}$, and $k c_{A}$ is the usual surface reaction rate (units of length per time), which depends only on the surface concentration $c_{A}$. As will be shown in Section 4, this leads to a well-defined continuum limit for the evolution of total fluid reactant mass, where the results are independent of both the discretization length $\ell_{d}$ and the subscale length $\ell_{s}$. Similarly, we assume here that molecular-scale attachment/detachment at the interface is fast compared to diffusion near the interface, so that it can be considered instantaneous. Specifically, this corresponds to assuming that $\tau_{a} / \tau_{d}$, where $\tau_{a}$ is the average duration of an attachment event and $\tau_{d}=\ell_{d}^{2} /(2 D)$ is the diffusion time associated with the discretization lengthscale, remains small. We note that the concept of scale separation, where a continuum limit is taken while maintaining a subscale length or timescale small, is commonly employed in volume averaging, see, e.g., [66].

Numerically, we implement these dynamics using particle tracking random walk (PTRW) simulations, which discretize the Langevin equation (1) (see, e.g., [61, 62] and Appendix A for further details). If a fluid-reactant particle is in the reactive region during a time step of duration $\Delta t$, its mass evolves according to

$$
M_{p}(t+\Delta t)=M_{p}(t) e^{-k_{d} \Delta t}
$$

Otherwise, if the particle is farther from the interface, no reaction occurs.

\section{Inter-reaction times and the chemical continuous time random walk}

In this section, we provide a brief description of the chCTRW framework and the associated concept of inter-reaction times, which will be used in what follows to obtain a quantitative description of total fluid reactant mass. In this framework, the inter-reaction time is the sum of the delay time due to transport limitations and the intrinsic reaction time necessary for the reaction 
(a)
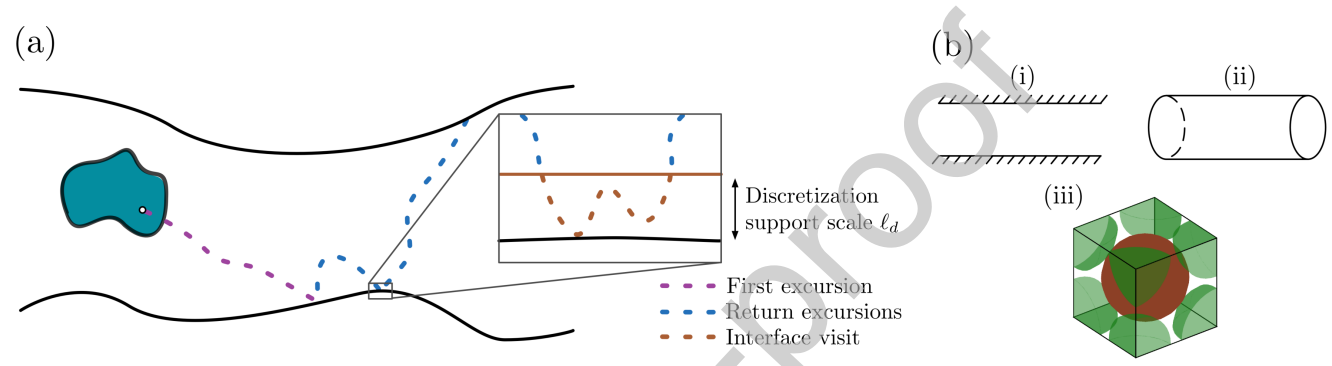

(iii)

Figure 1: (a): Illustration of the concepts of first passage, return, and interface visit times. The overall fluid-solid reaction rate is decreased due to the time spent by the fluid reactants away from the interface. Points in the initial condition (cyan blob) take different paths towards the interface (purple line). After the interface is reached for the first time, additional excursions away from and back to the interface (blue lines) also depend on medium geometry and transport. Obtaining a consistent description of the effect of the excursion times on reaction slowdown requires resolving the return excursions and interface visits (orange line) at the support scale of a discretized transport description before taking the continuum limit. (b): We apply the general framework to analyze reaction dynamics under advective diffusive transport in two- and three dimensional media: (i) Laminar flow in a straight 2D channel; (ii) Laminar flow in a cylindrical 3D channel; and (iii) Stokes flow in a body centered cubic beadpack, composed of a periodic array of the conventional cubic cells shown (the spherical solid bead at the cell center is shown in red, and the corner beads in green). 
to occur under well-mixed conditions. For the present application, these correspond to the times between visits of the particle to the reactive region, and the time spent in the reactive region. These concepts are illustrated in Fig. 1a. In the sections that follow, we will develop a theory of fluid-solid reaction under advective-diffusive transport, and apply it to analyze different two- and three-dimensional example media (Fig. 1b).

The chemical CTRW framework treats the inter-reaction time $\tau$, i.e., the time between successive reaction events, as a stochastic quantity incorporating variability from the chemical kinetics on the one hand, and transport and medium structure on the other. In what follows, we will obtain the PDF $\phi$ of inter-reaction times for our fluid-solid reaction. Disregarding for the moment the first excursion to the interface (purple excursion in Fig. 1), the total inter-reaction time in the chCTRW formulation can be written as [31]

$$
\tau=\tau_{r}+\tau_{g}\left(\tau_{r}\right)
$$

where $\tau_{r}$ is the time it would take for a reaction to occur if a particle were confined to the reactive region, and $\tau_{g}$ is the additional time spent in excursions away from the latter (blue excursions in Fig. 1). The total time $\tau_{r}$ that must be spent in the reactive region before the next reaction event impacts the delay time $\tau_{g}$, because a longer $\tau_{r}$ typically requires more visits to the reactive region (corresponding to orange excursions in Fig. 1), punctuated by excursions.

When small numbers of reactant particles (molecules) are considered, the inter-reaction time refers to the time between two successive reaction events between reactant molecules, allowing for capturing fluctuations due to finite particle numbers [28]. Recall that here we consider Lagrangian particles representing a certain amount of fluid reactant mass undergoing continuous advection-diffusion, and corresponding to a macroscopic number of molecules. In that case, fluctuations due to molecule numbers are not significant $[28,31]$. For conceptual and computational reasons, it is then more convenient to consider a fixed number of Lagrangian particles whose masses evolve in time [67]. In this case, the rate equation (3) for the evolution of the mass carried by a Lagrangian particle within the reactive region corresponds to a constant reaction rate $k_{d}=k c_{A} / \ell_{d}$ (with units of inverse time). According to the classical well-mixed theory for stochastic inter-reaction times, this reaction rate may be interpreted as a constant probability of reaction per unit time, which translates into an exponential inter-reaction time distribution [28]. Specifically, the probability that $\tau_{r}$ takes a value in $[u, u+d u)$ is 
given by $\phi_{r}(u) d u$, such that its probability density function (PDF) is given by

$$
\phi_{r}(u)=k_{d} e^{-k_{d} u} .
$$

For a given $\tau_{r}=u, \tau_{g}(u)$ is also a random variable, whose PDF $\phi_{g}(\cdot ; u)$ reflects the underlying medium heterogeneity and the stochasticity inherent in diffusive motion. In the next section, we will explicitly relate this PDF to the statistics of excursions away from the interface. As we will see, although $\tau_{r}$ and $\tau_{g}$ are discretization-dependent, $\tau$ is well defined in the continuum limit.

The impact of delay due to return excursions on reaction dynamics is captured by a memory function, given in terms of the PDF $\phi$ of inter-reaction times $\tau$ as [31]

$$
\tilde{K}_{\phi}(\lambda)=\frac{\lambda \tilde{\phi}(\lambda)}{1-\tilde{\phi}(\lambda)}
$$

Here and throughout, we denote Laplace transforms (with respect to time) by a tilde and the corresponding Laplace variable by $\lambda$. For the $A_{F}+B_{S} \rightarrow B_{S}$ reaction considered here, the total mass obeys

$$
\frac{d M_{\phi}}{d t}=-K_{\phi} * M_{\phi}
$$

where $*$ denotes the convolution product, $\left(K_{\phi} * M_{\phi}\right)(t)=\int_{0}^{\infty} d t^{\prime} K_{\phi}(t) M_{\phi}(t-$ $\left.t^{\prime}\right)$, and the subscript $\phi$ in the total mass indicates that the first delay time to reach the interface (see Fig. 1) has not yet been considered, so that these dynamics incorporate the impact of the inter-reaction time PDF $\phi$ only. This equation may be seen as a generalized rate law governing the time evolution of the total mass under the impact of reaction delay caused by transport limitations. In contrast to the classical well-mixed rate laws, where reaction rates depend only on the current mass in a batch reactor, the presence of the convolution with a memory function renders this equation integro-differential. Physically, this arises because the reaction rate at a given time depends on past history through the statistics of past excursions. Excursions away from and back to the reactive interface, controlled by transport and medium geometry, take the form of reaction delays, which lead to memory effects in the large-scale mass dynamics. Broad distributions of excursion times translate into long-range memory effects.

Applying these results to fluid-solid reactions requires relating the interreaction times to the statistics of excursions away from and back to the solid 
interface, as well as including the role of the first excursion to the interface (Fig. 1), representing the impact of the initial condition. This is the subject of the next section.

\section{Mass dynamics and inter-reaction times}

In this section, we first quantify the impact of diffusion near the reactive interface on the distribution of return times to the reactive region and the duration of each visit. Based on these concepts and the first passage time to the interface from the initial condition, we then obtain the inter-reaction times and the evolution of total fluid reactant mass based on the chCTRW formulation of the previous section.

\subsection{Return and visit times}

The dominant transport mechanism that controls local reactant mass flux towards the interface is diffusive, because there is no fluid flow into or out of the solid phase. We assume the interface to be locally flat at the scale of the transport model, so that, close to the interface, it is sufficient to consider one-dimensional diffusion in the transverse direction. We note that this local flatness assumption may be inappropriate in some systems, such as rough fractures, where the surface may exhibit fractal (i.e., self-similar across scales) properties (see, e.g., [68]). Such pronounced surface roughness is known to impact reaction rates [13]. A one-dimensional conceptualization of diffusion perpendicular to the surface may then be inaccurate, and more detailed modeling of transport and reaction near the interface may be necessary. This scenario, although important, is beyond the scope of the present work, and we do not explore it further here.

For a diffusing particle, characterized by the diffusion coefficient $D$, in an unbounded domain in one dimension, the PDF of the time to first reach a target located at a distance $\ell$ to one side of the initial position is given by the Lévy 1/2-stable density (see, e.g., [69]),

$$
\psi_{D}(t ; \ell)=\frac{\ell}{\sqrt{4 \pi D t^{3}}} e^{-\frac{\ell^{2}}{4 D t}}
$$

That is, $\psi_{D}(t ; \ell) d t$ is the probability that the first passage time to the target is in the interval $[t, t+d t)$. We have

$$
\tilde{\psi}_{D}(\lambda ; \ell)=e^{-\sqrt{\ell^{2} \lambda / D}} .
$$


In order to apply the chCTRW formulation, we require the time to return to a target, which sets the time between successive visits to the reactive interface, and therefore controls the inter-reaction times (see Fig. 1). This concept must be treated with care, because a particle undergoing continuous diffusive motion in one dimension crosses its original position infinitely many times in any given finite time interval. This is reflected in the fact that the limit $\ell \rightarrow 0$ of $\psi_{D}(t ; \ell)$ is not well define (see also [38, 40,41, 70]). To avoid this problem, we will obtain the inter-reaction times in the scaling limit of an appropriate discretization, associated with the support scale $\ell_{d}$ (see Section 2). Consider a regular one-dimensional discretization into intervals, or cells, of equal length $\ell_{d}$. The return times to the interface associated with the discretization are then the first passage times to the center of the cell touching the interface, from the center of the adjacent cell. The cell centers are a distance $\ell_{d}$ apart, so that the corresponding first passage time $\mathrm{PDF}$ is given by $\psi_{d}(\cdot)=\psi_{D}\left(\cdot ; \ell_{d}\right)$. Note that the same result is obtained by considering the first passage times to a distance $\ell_{d}$ from the interface, starting from a distance $2 \ell_{d}$. This is convenient for numerical determination from particle tracking simulations where particles can be placed at distance $2 \ell_{d}$ from the interface and the first passage time determined as the time when distance $\ell_{d}$ from the interface is crossed.

Before proceeding, we may relax the assumption of an unbounded domain, and the requirement of one-dimensional diffusive transport far from the reactive region. First, we denote the timescale associated with the discretization support scale $\ell_{d}$ by

$$
\tau_{d}=\frac{\ell_{d}^{2}}{2 D} .
$$

In order to allow for different effects away from the interface while retaining the diffusive behavior near it, we write the return time PDF in the form

$$
\tilde{\psi}_{d}(\lambda)=\tilde{\psi}_{D}\left(\lambda ; \ell_{d}\right)=e^{-\tilde{f}(\lambda) \sqrt{2 \tau_{d} \lambda}},
$$

see Eq. (10). The discretized description can only resolve times $t \gg \tau_{d}$, corresponding to $\lambda \ll 1 / \tau_{d}$. Thus, with a view to taking the continuum limit, it is sufficient to consider the limit of $\lambda \ll 1 / \tau_{d}$, corresponding for a given discretization to times large compared to the discretization time. We then find

$$
\tilde{\psi}_{d}(\lambda) \approx 1-\tilde{f}(\lambda) \sqrt{2 \tau_{d} \lambda} .
$$


It is important to note that this and similar results below do not represent late-time expansions, but rather lead to results valid for all times (or all $\lambda$ ) in the continuum limit $\ell_{d} \rightarrow 0$. In other words, finite- $\ell_{d}$ effects are a product of the discretization, which disappear in the continuum-limit description of total mass obtained in what follows.

The form factor $\tilde{f}(\lambda)$, which depends on the geometry of the domain and the transport mechanisms involved up to the timescale $1 / \lambda$, must approach unity for large $\lambda$, so that the behavior of the return time is dominated by the $\sqrt{2 \tau_{d} \lambda}$ term characterizing the diffusive behavior near the fluid-solid interface at short times. For small $\lambda$, corresponding to large times, $\tilde{f}(\lambda)$ encodes information about transport excursions far from the reactive region. As before, in an arbitrarily small time interval, continuous diffusion in one dimension crosses the origin infinitely many times. The factor $\sqrt{2 \tau_{d} \lambda}$, which approaches zero as $\ell_{d} \rightarrow 0$, captures the resulting singular character of the return time distribution in this limit, whereas the factor $\tilde{f}(\lambda)$, whose departure from unity represents additional effects from transport excursions unrelated to the discretization, remains finite and nonzero in the continuum limit.

Let $\ell_{c}$ be a characteristic lengthscale of the medium, such as the average pore size. Note that the choice of $\ell_{c}$ is arbitrary and simply provides a reference scale based on which nondimensional quantities characterizing the relationship between reaction, diffusion, and advection processes will be introduced below. We denote the corresponding diffusion time as

$$
\tau_{D}=\frac{\ell_{c}^{2}}{2 D}
$$

and define the Damköhler number

$$
\mathrm{Da}=\frac{k c_{A} \tau_{D}}{\ell_{c}} .
$$

The latter quantifies the relative importance of reaction and diffusive transport at this scale. It is convenient to define the rescaled return time tail probability

$$
g(t)=\frac{\ell_{c} \Psi_{d}(t)}{2 \tau_{D} \ell_{d}},
$$

where

$$
\Psi_{d}(t)=\int_{t}^{\infty} d t^{\prime} \psi_{d}\left(t^{\prime}\right)
$$


is the probability that the return times are greater than a given time $t$. In terms of the rescaled tail probability, the return times obey

$$
\tilde{\psi}_{d}(\lambda) \approx e^{-2 \tau_{D} \lambda \tilde{g}(\lambda) \ell_{d} / \ell_{c}},
$$

so that, comparing to Eq. (12), we have the relation $\tilde{g}(\lambda)=\tilde{f}(\lambda) / \sqrt{2 \tau_{D} \lambda}$. Since $\tilde{f}(\lambda)$ remains finite and nonzero in the limit $\ell_{d} \rightarrow 0$, so does $\tilde{g}(\lambda)$. Note that for large $\lambda$ we have $\tilde{g}(\lambda) \approx 1 / \sqrt{2 \tau_{D} \lambda}$, because $\tilde{f}(\lambda) \approx 1$ as discussed above. For small $\lambda$, corresponding to large times, $\tilde{g}(\lambda)$ again encodes the behavior of excursions away from the interface. These quantities are obtained analytically for the example of diffusion in a bounded one-dimensional domain in Section 5.

Note that the statistics of the excursions back to an arbitrary point along the interface may differ depending on the starting point, corresponding to different form factors and rescaled tail probabilities depending on the latter. Here, in order to obtain a stationary description where successive return times to the wall have the same statistics, we treat any such variability statistically. That is, the same rescaled tail probability is used to characterize the return time statistics associated with each point on the interface and at all times. In other words, this method disregards possible non-stationarity of the return times, as well as possible correlations between subsequent return times arising from different transport and geometry properties at different interface points. Nonetheless, we believe such a statistical description to be appropriate, as long as the flow is statistically stationary and the structural characteristics of the medium are statistically homogeneous within the region being considered. Note that this assumption is similar to that employed in the standard CTRW for transport [32], where variability in particle jump sizes and/or transit times is treated stochastically but assumed statistically homogeneous and stationary, that is, the PDFs associated with these quantities do not depend on the current time or particle position. In Section 6, we show that this approach leads to accurate predictions of mass dynamics in a regular beadpack, despite different points on the bead surfaces having different characteristics in terms of the distance to other nearby surface points.

Next, we turn to the statistics of the time spent in the reactive region in each visit. As shown in Appendix B, the corresponding PDF is given by

$$
\psi_{v}(t)=\frac{e^{-\frac{t}{2 \tau_{d}}}}{2 \tau_{d}}
$$


so that the single-visit times are exponentially distributed with mean $2 \tau_{d}$.

\subsection{Inter-reaction times}

We are now in a position to compute the PDF of global delay times $\tau_{g}$, which will allow us to obtain the PDF $\phi$ of the inter-reaction time $\tau$, see Eq. (5). Given a well-mixed reactive time $\tau_{r}=u$, the delay time due to excursions away from the reactive region is given by

$$
\tau_{g}(u)=\sum_{i=1}^{N_{U}(u)} W_{i},
$$

where the $W_{i}$ are independent and identically distributed return times with PDF $\psi_{d}$ and $N_{U}(u)$ is the (random) number of visits to the reactive region given well-mixed reactive time $u$. For sufficiently fine discretization, the residence times per visit to the reactive region are approximately exponential with mean $2 \tau_{d}$, Eq. (19). This implies that $N_{U}(u)$ is approximately Poisson with mean $u /\left(2 \tau_{d}\right)$ (see, e.g., [63]). Thus, the global delay (20) is a compound Poisson process, so that its PDF has Laplace transform [72-74]

$$
\tilde{\psi}_{g}(\lambda ; u)=\exp \left[-\frac{\lambda \tilde{\Psi}_{d}(\lambda) u}{2 \tau_{d}}\right] .
$$

The PDF $\phi$ of the total inter-reaction time $\tau$ resulting from a well-mixed reaction time $\tau_{r}$ with PDF given by Eq. (6) and a compound Poisson delay has been obtained in [31]. With the form (21) of the delay PDF, it reads

$$
\tilde{\phi}(\lambda)=\tilde{\phi}_{r}\left[\lambda\left(1+\frac{\tilde{\Psi}_{d}(\lambda)}{2 \tau_{d}}\right)\right] .
$$

In the continuum limit $\ell_{d} \rightarrow 0$, using Eqs. (6) and (12), we obtain

$$
\tilde{\phi}(\lambda)=\frac{\mathrm{Da}}{\mathrm{Da}+\tau_{D} \lambda \tilde{g}(\lambda)} .
$$

In this form, the inter-reaction times are manifestly well defined in the continuum limit. The effect of diffusion near the interface is implicit in the form of Eq. (23), and additional effects arising from domain geometry and/or velocity variability are encoded in the rescaled tail probability $g$, see Eq. (16). 


\subsection{Dynamics of reactant mass}

The chCTRW formulation can now be used to obtain the evolution of total mass given the inter-reaction time PDF together with the additional delay time to first reach the interface (see Fig. 1). This delay is distributed according to a PDF $\psi_{0}$, which depends on the transport mechanism and the initial reactant distribution. Defining

$$
\Psi_{0}(t)=\int_{t}^{\infty} d t^{\prime} \psi_{0}\left(t^{\prime}\right),
$$

the tail probability of the first passage time to the interface, we obtain, as shown in Appendix C,

$$
\frac{d M}{d t}=-K_{\phi} *\left(M-M_{0} \Psi_{0}\right) .
$$

This generalized, integro-differential ratelaw encodes the impact of transport and heterogeneity on the effective reaction kinetics through the inter-reaction times and the first passage time to the interface. In the standard CTRW description of transport, advection-dispersion at the small scales in the presence of heterogeneity leads to the emergence of memory kernels at larger scales in the advection-dispersion equation, accounting for statistical variability in particle jump sizes and/or transit times [32]. Analogously, the chCTRW leads to a description of total mass which incorporates the impact of transport limitations through a memory kernel representing statistical variability in first passage and inter-reaction times.

Taking the Laplace transform of Eq. (25), we obtain the Laplace-space solution for the evolution of total mass as

$$
\frac{\tilde{M}(\lambda)}{M_{0}}=\frac{\operatorname{Da} \tilde{\Psi}_{0}(\lambda)+\tau_{D} \tilde{g}(\lambda)}{\operatorname{Da}+\tau_{D} \lambda \tilde{g}(\lambda)} .
$$

While the number of visits to the reactive region within a given time window increases when the discretization is refined, the residence time associated with each visit decreases accordingly, and the total mass change due to reaction in the actual, subscale reactive region is well defined. These results connect the effect of transport limitations on mass dynamics to the inter-reaction times of the chCTRW under a transport mechanism for which mass exchange near a locally-flat interface is controlled by diffusion. In particular, this is 
the case for advection-diffusion: since there is no flux into or out of the solid interface, diffusion always dominates returns at sufficiently short times, when particles are close to the interface. This fact is incorporated into the form of Eq. (26). The rescaled tail probability $\tilde{g}(\lambda)$ captures further effects controlling the statistical variability of the excursion times, such as transverse velocity variations across the domain and the size of the latter, as will be discussed in more detail and illustrated in the following sections. Note that this description does not require zero- or low-velocity regions to occur only near the interface. The presence of stagnation regions affects $\tilde{g}(\lambda)$, and may lead to broader variability of the return times and therefore of the interreaction times.

We note that Eq. (26) can also be obtained in terms of the statistics of times spent near the interface, rather than the statistics of the inter-reaction times. This alternative formulation is discussed in Appendix D.

\section{Reactive transport in a $2 \mathrm{D}$ channel}

In order to illustrate the results of the previous section, we consider first the simple example of transport in an infinite two-dimensional channel with stratified flow and reactive walls (Fig. 1b, (i)), for which fully-analytical results can be obtained in Laplace space. We take the characteristic length $\ell_{c}$ as the channel half-width.

\subsection{Analytical first passage/return times and mass dynamics}

The distribution of times to reach either wall from a given point along the channel cross-section depends in this case only on transverse diffusion and not on the velocity profile. It is thus sufficient to consider one-dimensional diffusive transport along the channel cross-section. The corresponding PDF in a bounded domain of size $L$, starting from a distance $\ell$ from one wall, has Laplace transform [71]

$$
\tilde{\psi}(\lambda ; \ell, L)=\frac{\operatorname{csch}\left[\sqrt{\ell^{2} \lambda / D}\right]+\operatorname{csch}\left[\sqrt{(L-\ell)^{2} \lambda / D}\right]}{\operatorname{coth}\left[\sqrt{\ell^{2} \lambda / D}\right]+\operatorname{coth}\left[\sqrt{(L-\ell)^{2} \lambda / D}\right]} .
$$

In order to determine the first return time PDF, in the sense discussed in the previous section, we take $\ell=\ell_{d}$ and $L=2 \ell_{c}$. In preparation for taking the continuum limit $\ell_{d} \rightarrow 0$, we expand for small $\ell_{d} \ll \ell_{c}$ to obtain

$$
\tilde{\psi}_{d}(\lambda) \approx \exp \left[-\tanh \left(\sqrt{2 \tau_{D} \lambda}\right) \sqrt{2 \tau_{d} \lambda}\right] \approx 1-\tanh \left(\sqrt{2 \tau_{D} \lambda}\right) \sqrt{2 \tau_{d} \lambda},
$$


which, according to Eqs. (12) and (18), corresponds to the form factor and rescaled tail probability

$$
\tilde{f}(\lambda)=\tanh \left(\sqrt{2 \tau_{D} \lambda}\right), \quad \quad \tilde{g}(\lambda)=\frac{\tanh \left(\sqrt{2 \tau_{D} \lambda}\right)}{\sqrt{2 \tau_{D} \lambda}} .
$$

Substituting $\tilde{g}(\lambda)$ in Eq. (26), the total mass as a function of time has Laplace transform

$$
\frac{\tilde{M}(\lambda)}{M_{0}}=\frac{2 \mathrm{Da} \tilde{\Psi}_{0}(\lambda)+\tanh \left(\sqrt{2 \tau_{D} \lambda}\right) \sqrt{2 \tau_{D} / \lambda}}{2 \mathrm{Da}+\tanh \left(\sqrt{2 \tau_{D} \lambda}\right) \sqrt{2 \tau_{D} \lambda}} .
$$

For small Da, we can expand this result for small $\lambda \ll 1 / \tau_{D}$ (time $t \gg$ $\left.1 / \tau_{D}\right)$ before appreciable reaction occurs, and we obtain

$$
\tilde{M}(\lambda) \approx \frac{M_{0}}{\mathrm{Da} / \tau_{D}+\lambda}, \quad \quad M(t) \approx M_{0} e^{-\mathrm{Da} t / \tau_{D}} .
$$

Hence, the reaction kinetics correspond to a fully-mixed cross-section. This arises because, for slow reaction compared to the diffusive time scale, diffusion homogenizes the fluid reactant to a mass per cross-section length $1 /\left(2 \ell_{c}\right)$ before appreciable reaction occurs. Since there are two reaction interfaces, the fraction of mass in the reactive region is $2 \ell_{d} /\left(2 \ell_{c}\right)=\ell_{d} / \ell_{c}$. The effective reaction rate is thus $k_{d} \ell_{d} / \ell_{c}=\mathrm{Da} / \tau_{D}=k c_{A} / \ell_{c}$, independent of the diffusion coefficient.

For large Da and an initial condition not fully concentrated at the channel walls, so that $\tilde{\Psi}_{0}(\lambda) \neq 0$, the initial condition dominates, and we have

$$
M(t) \approx M_{0} \Psi_{0}(t)
$$

This result can be interpreted as follows. In the limit of fast reaction, the surviving mass is that which has never reached the channel walls. The fraction of the initial mass that has not reached the walls is given by the probability $\Psi_{0}(t)$ that the first passage time to the walls from the initial position is greater than $t$.

In the particular case where all the mass starts at the channel walls, $\tilde{\Psi}_{0}(\lambda)=0$, the dynamics are fully controlled by the return times. For large Da and $\lambda \gtrsim 1 / \tau_{D}$ (early times), we obtain

$$
\tilde{M}(\lambda) \approx \frac{M_{0}}{\mathrm{Da}} \sqrt{\frac{\tau_{D}}{2 \lambda}}, \quad M(t) \approx \frac{M_{0}}{\mathrm{Da}} \sqrt{\frac{\tau_{D}}{2 \pi t}} .
$$


For $\lambda \lesssim 1 / \tau_{D}$ (late times), we find

$$
\tilde{M}(\lambda) \approx \frac{M_{0}}{\mathrm{Da}} \frac{\tau_{D}}{1+2 \tau_{D} \lambda / 3}, \quad M(t) \approx \frac{3 M_{0}}{2 \mathrm{Da}} e^{-\frac{3 t}{2 \tau_{D}}}
$$

This means that diffusive excursions far from the walls control reaction until the channel cross-section is homogenized by diffusion, after which we recover exponential behavior, but diffusion- rather than reaction-limited: the exponent of the exponential decay in (34) depends only on the diffusion coefficient, while that of (31) depends only on the reaction rate.

While the form of the mass dynamics, Eq. (30), is flow-profile-independent, the actual mass evolution can depend on the profile through the initial condition, such as for a flux-weighted condition. In the low-Da limit discussed above, the initial delay does not play a relevant role and this dependence disappears. For definiteness, consider two-dimensional Poiseuille flow, with the velocity profile

$$
v(y)=v_{M}\left(1-\frac{y^{2}}{\ell_{c}^{2}}\right)
$$

for $y \in\left[-\ell_{c}, \ell_{c}\right]$ along the one-dimensional cross-section, with $v_{M}$ the maximum velocity, attained at the channel center, $y=0$. The corresponding Eulerian mean velocity is given by $\bar{v}=2 v_{M} / 3$.

Different initial conditions affect the initial first passage time until the interface is first reached. We consider four examples of instantaneous injection, with the fluid reactant mass placed: (a) at the middle of the channel, (b) homogeneously along the channel cross section, (c) flux-weighted along the channel width, and (d) at the channel walls; the Laplace transforms of the corresponding first passage time PDFs $\psi_{0}$ are given in Appendix E.

\subsection{Numerical simulations of reactive transport}

We performed PTRW simulations of the two-dimensional reactive transport problem, as described in Section 2 and Appendix A. We nondimensionalize distances by $\ell_{c}$, time by the diffusion time $\tau_{D}$, and mass by the initial mass $M_{0}$. Note that, numerically, this can be conveniently achieved by setting the diffusion coefficient to $D=1 / 2$, the channel half-width to $\ell_{c}=1$, and the initial mass to $M_{0}=1$, in arbitrary units. Each of $N$ particles initially carries a fraction $M_{0} / N$ of the initial mass. In order to obtain good statistics, the number $N$ of particles used must be such as to permit resolving the transverse direction to within the discretization length, meaning 


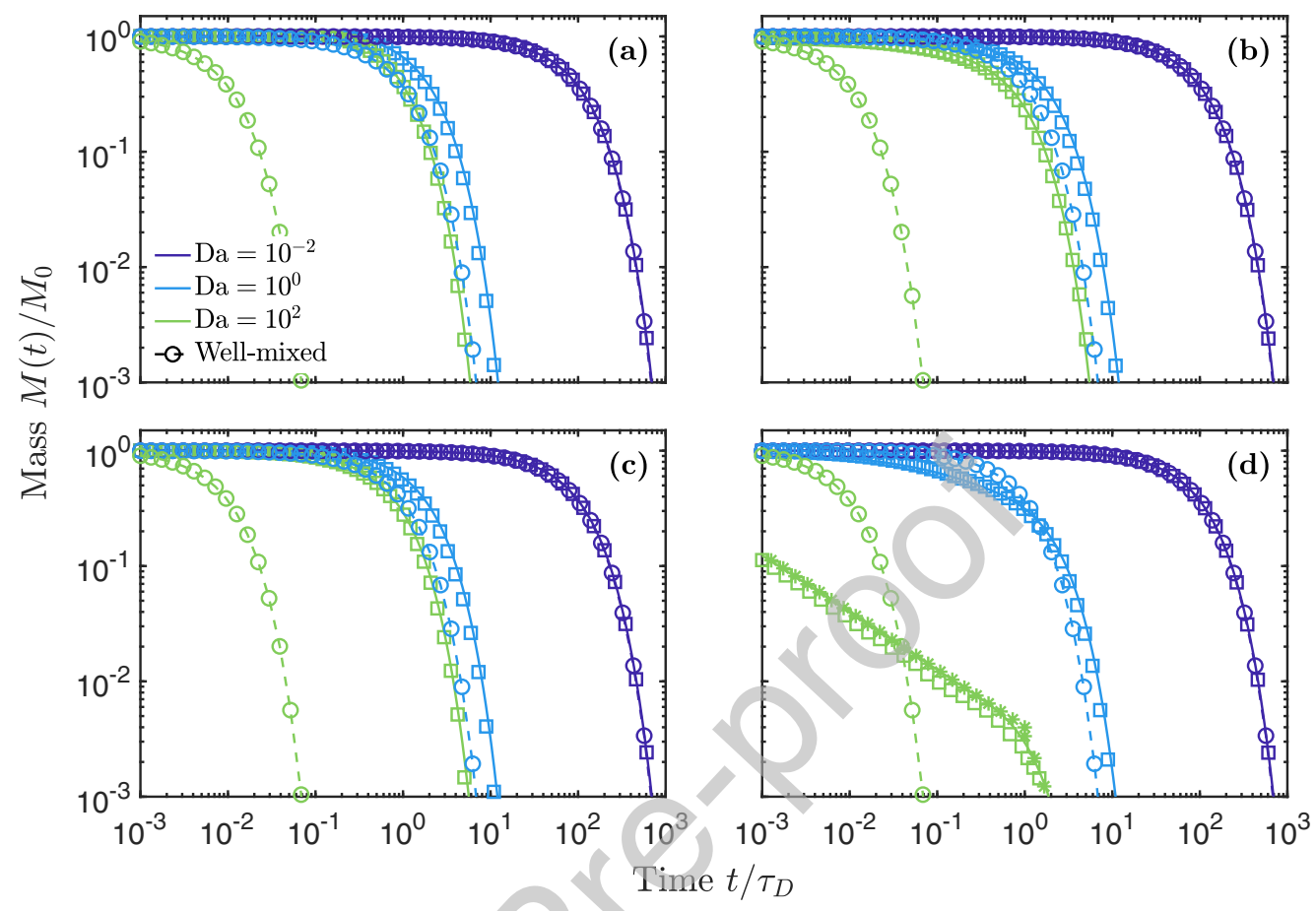

Figure 2: Reactant mass as a function of time for Poiseuille flow in a two-dimensional channel, for different Damköhler numbers Da (colors) and initial conditions (a)-(d). Solid lines show numerical inversion of Eq. (30) and square markers represent reactive PTRW simulations. Circles correspond to the analytical solution (31) for a well-mixed crosssection. (a): Channel middle, (b): Uniform. (c): Flux-weighted. (d): Channel walls. Asterisks in (d) correspond to the analytical solutions (33) for $t / \tau_{D} \leqslant 1$ and (34) for $t / \tau_{D}>1$. PTRW simulations for $\mathrm{Da}=10^{0}, 10^{2}$ use a time step $\Delta t=10^{-4} \tau_{D}$ and $N=10^{3}$ particles. For Da $=10^{2}, N=10^{4}$, with $\Delta t=10^{-6} \tau_{D}$ for (a)-(c) and $\Delta t=10^{-7} \tau_{D}$ for (d).

$N \gtrsim \ell_{c} / \ell_{d}=\sqrt{\tau_{D} / \tau d}$. Aside from setting the spatial resolution through $\ell_{d}$, the time step must be sufficiently small to resolve reaction. Since the average residence time per visit to the reactive region is $2 \tau_{d}$, this requires $2 k_{d} \tau_{d} \lesssim 1$. Note that, even though the initial condition (c) depends on the velocity profile, the first passage time (E.3) is independent of the mean velocity, so that we can arbitrarily set $\bar{v}=1$.

The evolution of total mass under the different initial conditions (a)-(d) is shown in Fig. 2. Good agreement is observed between simulations and numerical inversion of Eq. (30). Note how the Da $=10^{-2}$ case is identical for all initial conditions; it corresponds to the low-Da limit, Eq. (31), characterized 

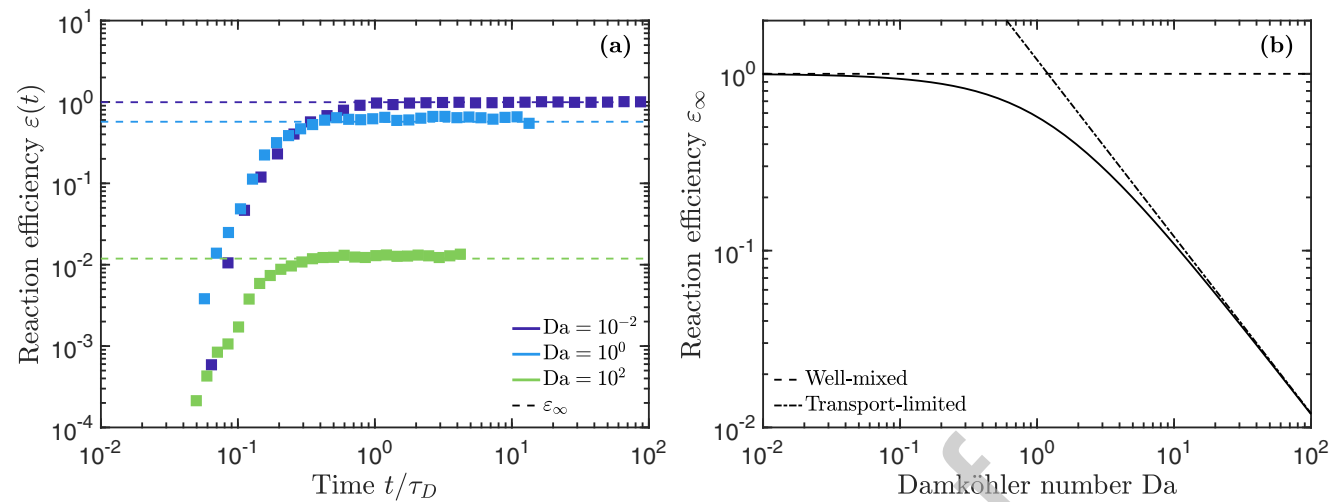

Figure 3: Reaction efficiency $\varepsilon(t)$, comparing the observed overall reaction rate as a function of time to the rate for a well-mixed two-dimensional channel cross-section. The initial condition is concentrated at the middle of the channel. (a): Reaction efficiency computed from the data in Figure 2a (symbols). The theoretical asymptotic value $\varepsilon_{\infty}$ is shown as a dashed line for each value of the Damköhler number Da. (b): Theoretical assymptotic reaction efficiency $\varepsilon_{\infty}$ as a function of Da. The well-mixed (low-Da) and fully transportedlimited (high-Da) limits are shown as dashed and dash-dotted lines, respectively.

by purely exponential decay and fully controlled by the reaction rate, i.e., independent of the diffusion coefficient. In (a)-(c), the Da $=10^{2}$ curves have converged to the Da-independent fast reaction limit, Eq. (32). This regime is the most affected by the initial condition, because it is controlled by the initial first passage time to the interface. However, the characteristic first passage times associated with conditions (a)-(c) are all on the order of the diffusion time $\tau_{D}$, and thus lead to qualitatively similar mass decay. For (d), the evolution of the total mass is fully controlled by the return times to the reactive region, Eqs. (33) and (34). The late-time behavior in this case is also exponential, but fully controlled by the diffusion coefficient, i.e., independent of the reaction rate constant $k$ and the solid-phase surface concentration $c_{A}$. Note how, for moderate to high Damköhler number, Da $\gtrsim 1$, the solution (31) corresponding to a well-mixed cross-section tends to overestimate reaction. This is the case even for the uniform initial condition (a), because fast consumption of solute at the interface inhibits transverse homogeneity. At early times in (d), reaction is faster than the well-mixed-channel prediction in these regimes due to solute starting in the reactive region, but slower at later times as the surviving solute explores the channel cross-section. 


\subsection{Reaction efficiency}

These results show that, while reaction rates tend to increase with the Damköhler number as would be expected, the reaction efficiency compared to a well-mixed channel cross-section characterized by the same Da decreases due to transport limitations. In order to better understand this phenomenon, consider the global, or effective, reaction rate

$$
k_{e}(t)=\left|\frac{d \ln M(t) / M_{0}}{d t}\right| .
$$

In other words, the effective rate $k_{e}(t)$ is defined so that $d M(t) / d t=-k_{e}(t) M(t)$. Note that, for pure exponential decay at constant rate, $M(t)=M_{0} \exp \left(-k_{e} t\right)$, this definition recovers $k_{e}(t)=k_{e}$ as expected. We define the reaction efficiency by comparing $k_{e}(t)$ to the reaction rate $k_{e}^{\mathrm{wm}}$ associated with well-mixed conditions in the domain,

$$
\varepsilon(t)=\frac{k_{e}(t)}{k_{e}^{\mathrm{wm}}} .
$$

According to Eq. (31), the well-mixed reaction rate for the two-dimensional channel is $k_{e}^{\mathrm{wm}}=\mathrm{Da} / \tau_{D}$. It is important to keep in mind the different physical meanings of the effective well-mixed rate $k_{e}^{\mathrm{wm}}$ and the thermodynamic well-mixed rate $k$ in Eq. (2). The latter, defined in units of inverse concentration per time, is the usual batch-reactor rate, which governs fully well-mixed reaction. The former, defined in units of inverse time, represents the effective reaction rate occurring when the (fluid-phase) domain is well-mixed. It is impacted by medium geometry, as it accounts for the fact that part of the fluid reactant remains far from the interface.

We now obtain a theoretical expression for the asymptotic rate $k_{e}^{\infty}=$ $\lim _{t \rightarrow \infty} k_{e}(t)$ and the corresponding asymptotic reaction efficiency $\varepsilon_{\infty}=k_{e}^{\infty} / k_{e}^{\mathrm{wm}}$. To this end, we introduce the mean first passage time to the wall $w_{0}$ and the corresponding second moment,

$$
w_{0}=\int_{0}^{\infty} d t t \psi_{0}(t), \quad s_{0}=\int_{0}^{\infty} d t t^{2} \psi_{0}(t) .
$$

We define also

$$
\alpha_{0}=\frac{s_{0}}{2 \tau_{D} w_{0}},
$$


which represents a dimensionless measure of the variability in the first passage times, compared to the diffusion time $\tau_{D}$. Expanding Eq. (30) for small $\lambda \lesssim 1 / \tau_{D}$, and inverting the Laplace transform, we find the late-time behavior

$$
\begin{aligned}
\frac{M(t)}{M_{0}} & \approx\left(w_{0}+\frac{\tau_{D}}{\mathrm{Da}}\right) k_{e}^{\infty} e^{-k_{e}^{\infty} t}, \\
k_{e}^{\infty} & =\frac{2 \mathrm{Da}\left(\mathrm{Da} w_{0}+\tau_{D}\right)}{\mathrm{Da}^{2} s_{0}+\operatorname{Da} \tau_{D}\left(2 w_{0}+4 \tau_{D} / 3\right)+2 \tau_{D}^{2}},
\end{aligned}
$$

so that

$$
\varepsilon_{\infty}=\left(1+\frac{\tau_{D}}{w_{0} \mathrm{Da}}\right)\left(1+\frac{2 \tau_{D}}{3 w_{0}}+\alpha_{0} \mathrm{Da}+\frac{\tau_{D}}{w_{0} \mathrm{Da}}\right)^{-1} .
$$

Thus, for small Da, we recover the well-mixed rate as predicted by Eq. (31), and $\varepsilon_{\infty} \approx 1$. For high Da and an initial condition not concentrated at the channel walls, the asymptotic reaction rate is limited by the first passage times to the interface, and $k_{e}^{\infty} \approx 1 /\left(\alpha_{0} \tau_{D}\right)$. For a given initial condition, this asymptotic rate cannot exceed a constant value. Since the well-mixed rate is linear in Da, the asymptotic efficiency $\varepsilon_{\infty} \approx 1 /\left(\alpha_{0} \mathrm{Da}\right)$ is inversely proportional to Da. In the special case of the initial condition at the channel walls, we recover Eq. (34), and $\varepsilon_{\infty} \approx 3 /(2 \mathrm{Da})$. In this case, reaction is limited by the return times to the wall, and the asymptotic efficiency remains inversely proportional to Da.

These results are illustrated for the case of a mid-channel injection, initial condition (a), in Fig. 3. From Eq. (E.1), we find in this case $w_{0}=\tau_{D}, s_{0}=$ $5 \tau_{D}^{2} / 3$, and $\alpha_{0}=5 / 6$. As predicted, the reaction rate becomes asymptotically constant for all Damköhler, corresponding to exponential decay. However, the reaction rate is initially variable as the fluid reactant explores the channel cross-section. For this choice of initial condition, the reaction efficiency is initially zero because the solute is far from the interface, and subsequently increases to the asymptotic value.

\section{Generalization to other media}

In this section, we will discuss the generalization of our approach to more complex scenarios, where fully analytical results for the inter-reaction times are not available. As long as diffusion is the dominant transport mechanism near the reactive interface, and the latter can be assumed flat at the scale 
of the transport model, Eq. (26) may be used to predict the time evolution of total mass for arbitrary Damköhler number, given knowledge of the tails $\Psi_{0}$ and $\Psi_{d}$ of the first passage and return times. In general, analytical expressions for these quantities are not available, and they must therefore be determined numerically. However, some general results about the mean return time, if it exists, may be obtained and employed to determine asymptotic reaction rates. Note that the existence of a mean inter-reaction time is directly related to the existence of a mean return time to the interface. If the excursions away from and back to the interface (see Fig. 1) have sufficiently heavy-tailed duration statistics, the chCTRW framework predicts a similar effect on reaction dynamics [31]. Such situations, which we do not consider further here but can be captured in our framework, can be important when modeling scenarios where solute may be retained in large regions of low velocity away from the reactive interface, or where the distribution of distances between separate reactive regions is very broad and not well characterized by a mean value [47].

\subsection{Mean return times and effective reaction rate}

The asymptotic reaction rate for stratified flow in a 2D channel was obtained in Section 5 , Eq. (41). In that case, the limit of low Da, corresponding to slow reaction, leads to an effective reaction rate of $\mathrm{Da} / \tau_{D}$, Eq. (31). We begin by generalizing this result by considering the mean return time to the interface,

$$
w_{d}=\int_{0}^{\infty} d t t \psi_{d}(t) .
$$

We have for the return time $\operatorname{PDF} \tilde{\psi}_{d}(\lambda) \approx 1-w_{d} \lambda$, for $\lambda \ll 1 / w_{d}$, so that the rescaled tail probability, Eq. (16), obeys

$$
\tilde{g}(0)=\int_{0}^{\infty} d t g(t)=\frac{w_{d} \ell_{c}}{2 \tau_{D} \ell_{d}} .
$$

Taylor-expanding Eq. (26) for small $\lambda$ and inverting the Laplace transform,

$$
M(t)=M_{0} e^{-k_{e} t},
$$

with the effective reaction rate

$$
k_{e}=\frac{2 \mathrm{Da} \ell_{d}}{w_{d} \ell_{c}} .
$$


In order to relate the mean return time $w_{d}$ to medium structure, we now determine the effective rate in a well-mixed domain. Consider that the solute is well-mixed over a region of volume $V$, within which the volume of the reactive region is $V_{d}$. The reactive region comprises a layer of height $\ell_{d}$ immediately adjacent to the interface, so that $V_{d} \approx A_{I} \ell_{d}$, where $A_{I}$ is the interface area. The mass in the reactive region is then $M(t) V_{d} / V$, and reacts at rate $k_{d}$, see Eq. (3), so that

$$
\frac{d M(t)}{d t}=-k_{e}^{\mathrm{wm}} M(t),
$$

with the well-mixed effective reaction rate given by

$$
k_{e}^{\mathrm{wm}}=\frac{V_{d}}{V} k_{d}=\frac{\rho \mathrm{Da}}{\tau_{D}},
$$

where we have introduced the dimensionless interface-extent coefficient

$$
\rho=\frac{A_{1} \ell_{c}}{V},
$$

encoding the amount of interface area per unit volume. Note that, if a representative elementary volume for interface area exists, and the solute is well-mixed over a larger volume, $\rho$ is given by this ratio over the representative volume. Note also that for an empty channel of cross-section $A$, we have

$$
\rho=\frac{\ell_{I} \ell_{c}}{A}
$$

where $\ell_{I}$ is the total interface length intersecting the cross-section. If the medium is statistically homogeneous along the flow direction, this formula may also be used to compute $\rho$ given information about the interface extent over a cross-section, taking $A$ as the fluid-phase area over the latter.

If the concentration becomes well-mixed due to transport before appreciable reaction occurs, the effective reaction rates associated with the return time and well-mixed-domain pictures must coincide, $k_{e}=k_{e}^{\mathrm{wm}}$. Using Eqs. (45) and (47), we find the mean return time and associated effective reaction rate as

$$
w_{d}=\frac{2 \tau_{D} \ell_{d}}{\rho \ell_{c}}, \quad \quad k_{e}=\frac{\rho \mathrm{Da}}{\tau_{D}} .
$$


Note that these results agree with the example considered in Section 5. In that case, $\ell_{I}=2, A=2 \ell_{c}, \rho=1$, and $k_{e}=\mathrm{Da} / \tau_{D}$. Note also that $w_{d}$ is independent of the Damköhler number, so that, according to Eq. (43),

$$
\tilde{g}(0)=\rho^{-1},
$$

independent of Da, so long as the transport mechanism leads to a wellmixed state in the absence of reaction. We note that, as before, for large Da (fast reaction) and an initial condition not concentrated along the interface, reaction is controlled by the first passage times to the interface, and $M(t)=$ $M_{0} \Psi_{0}(t)$. In that case, the reaction dynamics are thus controlled by the initial reactant distribution.

\subsection{Reaction efficiency}

We can now generalize the results for the asymptotic reaction efficiency. Using Eqs. (16) and (51), we find the small $-\lambda \lesssim 1 / \tau_{D}$ expansion for the rescaled tail probability:

$$
\tilde{g}(\lambda) \approx \rho^{-1}(1-\alpha \lambda / 2),
$$

where $\alpha$ is defined by

$$
\alpha=\frac{s_{d}}{2 \tau_{D} w_{d}}, \quad s_{d}=\int_{0}^{\infty} d t t^{2} \psi_{d}(t),
$$

quantifying the variability in the return times to the interface relative to the diffusion time. Using this result to expand Eq. (26) for $\lambda \lesssim 1 / \tau_{D}$, and inverting the Laplace transform, we find the late-time behavior

$$
\begin{aligned}
\frac{M(t)}{M_{0}} & \approx \frac{\rho \mathrm{Da} w_{0}+\tau_{D}}{\operatorname{Da}} k_{e}^{\infty} e^{-k_{e}^{\infty} t}, \\
k_{e}^{\infty} & =\frac{2 \rho \operatorname{Da}\left(\operatorname{Da} w_{0}+\tau_{D}\right)}{\rho^{2} \mathrm{Da}^{2} s_{0}+\rho \operatorname{Da} \tau_{D}\left(2 w_{0}+\alpha\right)+2 \tau_{D}^{2}} .
\end{aligned}
$$

We thus conclude that, as long as the transport mechanism leads to a wellmixed state in the absence of reaction, the reaction rate always asymptotes to a constant at late times, for arbitrary Da. Using Eqs. (37) and (47), the corresponding asymptotic reaction efficiency is

$$
\varepsilon_{\infty}=\left(1+\frac{\tau_{D}}{w_{0} \rho \mathrm{Da}}\right)\left(1+\alpha+\alpha_{0} \rho \mathrm{Da}+\frac{\tau_{D}}{w_{0} \rho \mathrm{Da}}\right)^{-1}
$$


see also Eqs. (38) and (39). For the 2D channel case, $\rho=1$ and $\alpha=2 / 3$ from Eq. (28), so that this result reduces to Eq. (41). In the low-Da limit of slow reaction, we recover Eq. (47) for the well-mixed reaction rate and obtain $\varepsilon_{\infty} \approx 1$ as expected. For high Da and an initial condition not concentrated at the channel walls, we have the initial-condition-limited results $k_{e}^{\infty} \approx 1 /\left(\alpha_{0} \tau_{D}\right)$ and $\varepsilon_{\infty} \approx 1 /\left(\alpha_{0} \rho \mathrm{Da}\right)$. In the special case of an initial condition distributed over the interface, for which $w_{0}=s_{0}=0$, we find $k_{e}^{\infty} \approx 1 /\left(\alpha \tau_{D}\right)$, emphasizing that in this case the transport limitations come into play through the return times rather than the first passage times. As before, $\varepsilon_{\infty} \approx 1 /(\alpha \rho \mathrm{Da})$ remains inversely proportional to Da.

\subsection{Mass dynamics from numerical first passage and return statistics}

According to Eq. (16), $\tilde{g}(\lambda)$ is proportional to $\tilde{\Psi}_{d}(\lambda)$. Thus, mass dynamics can be quantified through Eq. (26) by numerically sampling first passage and first return times in PTRW simulations of the transport. For a given initial condition, the Laplace transform $\tilde{\Psi}_{0}(\lambda)$ can be found directly from the fraction of first passage time samples to the interface above a given time. To determine the first return times associated with discretization length $\ell_{d}$ for a given medium and flow, we take a single-particle initial condition chosen uniformly randomly over the surface at (perpendicular) distance $2 \ell_{d}$ from the interface, corresponding to a distance $\ell_{d}$ from the reactive region. Once the reactive region is reached, we record the duration of the excursion, place the particle at the closest point at distance $2 \ell_{d}$ from the interface, and repeat the process for the next excursion a prescribed number of times. Note that, in the cases considered here, the system is asymptotically well-mixed over a representative region, so that different points along the interface are revisited with the same probability. This means that equivalent results would be obtained by considering the first passage time to within $\ell_{d}$ of the interface for a set of particles initially distributed uniformly over the latter.

A comparison of the rescaled tail probabilities $g(t)$ obtained in this manner for one-dimensional diffusion in a bounded domain of half-width $\ell_{c}$, along with the rescaled first return time $\mathrm{PDF} \psi_{d}(t) \ell_{c} / \ell_{d}$, with those obtained from numerical inversion of the analytical Laplace transform of $\tilde{\Psi}_{d}(\lambda)$, Eq. (27) with $\ell=\ell_{d}$ and $L=2 \ell_{c}$, is shown in Fig. 4 for two different values of $\ell_{d}$. We nondimensionalize distances by $\ell_{c}$ and times by $\tau_{D}$ as before. For $t \gg \Delta t$, the return time statistics converge to the same discretization-independent behavior. Above the discretization timescale $\tau_{d}$, the late-time scalings follow those of pure diffusion in an semi-infinite domain, Eq. (9), until the effect of 

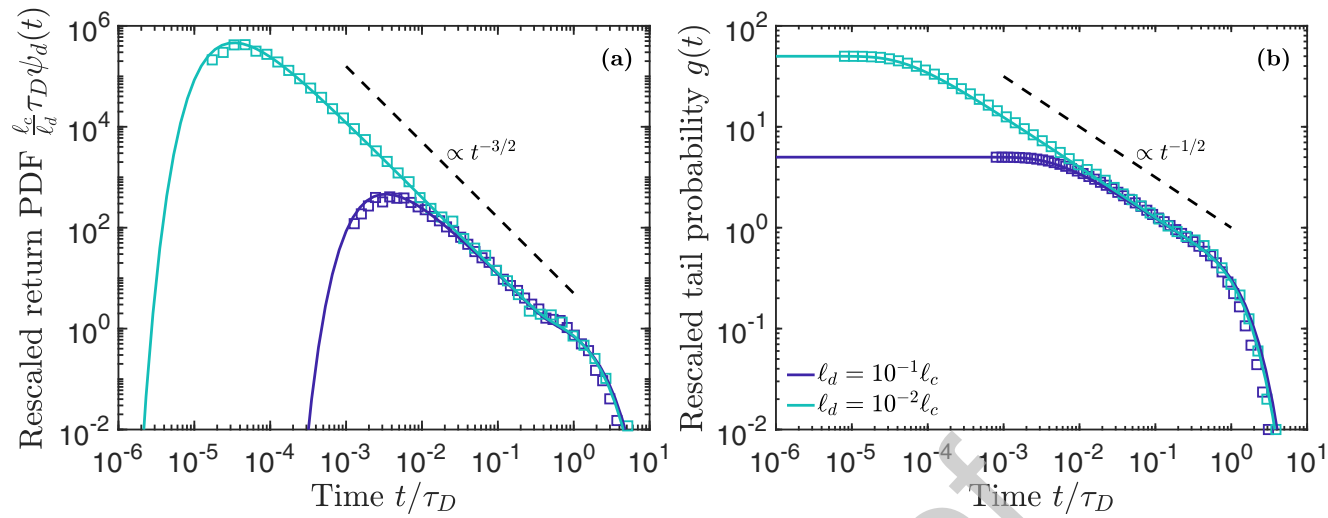

Figure 4: Rescaled first return time $\operatorname{PDF}$ (a) and return time tail probabilities (b) for one-dimensional diffusion in a bounded domain, obtained from $10^{4}$ return time samples from PTRW simulations (symbols) and numerical inversion according to the analytical Laplace transform (27) (lines). The large-time scalings associated with pure diffusion in a semi-infinite domain, which here hold below the characteristic diffusion time $\tau_{D}$ for sampling the full domain and above the discretization timescale $\tau_{d}=\ell_{d}^{2} /(2 D)=\tau_{D} \ell_{d}^{2} / \ell_{c}^{2}$, are shown as dashed lines.

the far boundary is felt, leading to a cutoff on a characteristic timescale of order $\tau_{D}$, corresponding to exploring the full domain.

\subsubsection{Reactive transport in a 3D channel}

As a first example to verify the results for the mass dynamics using numerical estimation of the first passage and return times, consider transport in a three-dimensional, cylindrical channel, with the characteristic length $\ell_{c}$ given by the channel radius (Fig. 1b, (ii)). We take a point injection at the center of the channel as the initial condition. As before, it suffices to consider diffusion along a cross-section in order to determine the evolution of total mass, independent of the flow field as long as it is assumed to be stratified. We again nondimensionalize distances by $\ell_{c}$, time by $\tau_{D}$, and initial mass by $M_{0}$.

The results obtained from numerical inversion of Eq. (26), given numerical determination of the first passage and return times, are in good agreement with reactive PTRW simulations, as shown in Fig. 5a. In this case, the crosssection area is given by $A=\pi \ell_{c}^{2}$, and the interface length intersecting a crosssection by $\ell_{I}=2 \pi \ell_{c}$, so that the interface-extent coefficient is $\rho=2$, Eq. (49). For $\mathrm{Da}=10^{-2}$, we find good agreement between the numerical simulations and exponential decay according to the effective well-mixed reaction rate, 

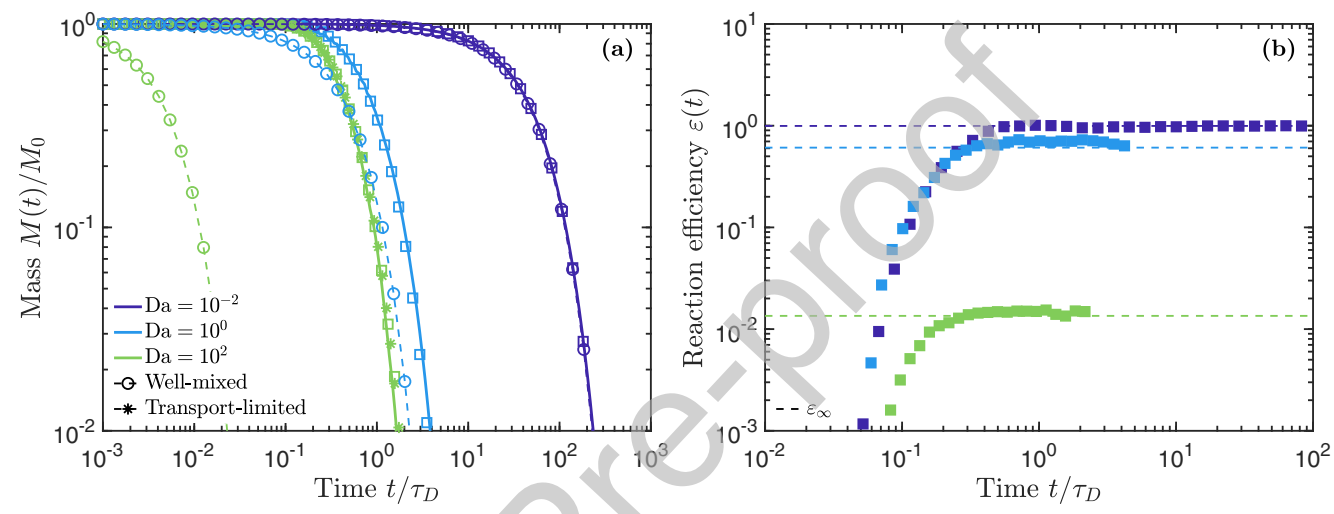

Figure 5: (a): Total mass as a function of time in a three-dimensional, cylindrical channel, for different Damköhler numbers Da (colors). The initial condition is concentrated at the channel center. Solid lines show numerical inversion of Eq. (30) given numerical computation of the first passage and return times, and square markers represent reactive PTRW simulations. Circles correspond to the analytical solution with the effective wellmixed reaction rate (45) for $\rho=2$. Asterisks represent the fully transport-limited solution $M(t)=M_{0} \Psi_{0}(t)$. First passage and return statistics are computed from $10^{4}$ samples, using a time step $\Delta t=10^{-6} \tau_{D}$. Reactive PTRW simulations use $\Delta t=10^{-4} \tau_{D}$ and $N=10^{3}$ particles for $\mathrm{Da}=10^{-2}, 10^{0}$, and $\Delta t=10^{-6} \tau_{D}$ and $N=10^{4}$ particles for $\mathrm{Da}=$ $10^{2}$. (b): For each value of Da, the reaction efficiency computed according to numerical differentiation of the data in (a) is shown as markers, and the theoretical asymptotic value $\varepsilon_{\infty}$, computed based on the same first passage and return data, is shown as a dashed line. 

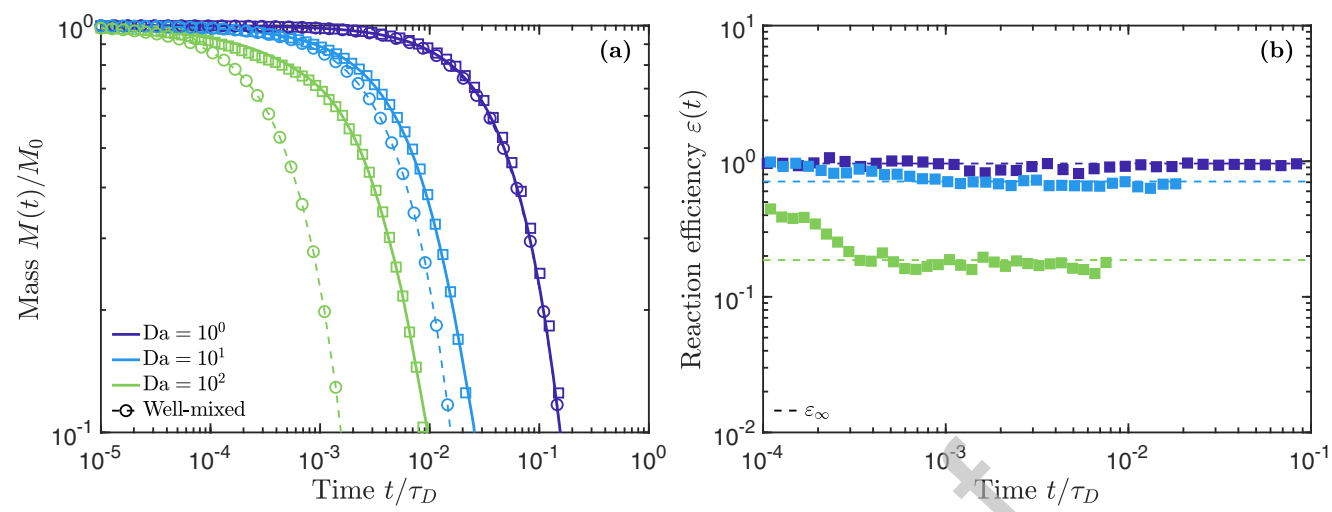

Figure 6: (a): Total mass as a function of time in a body centered cubic beadpack, for different Damköhler numbers Da (colors). The Péclet number is $\mathrm{Pe}=10^{3}$, and the initial condition is homogeneous in the conventional unit cell. Solid lines show numerical inversion of Eq. (30) given numerical computation of the first passage and return times, and square markers represent reactive PTRW simulations. Circles correspond to the analytical solution with the well-mixed reaction rate (45). First passage statistics are computed from $10^{4}$ samples and return statistics from $10^{5}$ samples, both using a time step $\Delta t=10^{-7} \tau_{D}$. Reactive PTRW simulations use $N=10^{3}$ particles, with $\Delta t=10^{-7} \tau_{D}$ for $\mathrm{Da}=10^{0}, 10^{1}$ and $\Delta t=10^{-8} \tau_{D}$ for $\mathrm{Da}=10^{2}$. (b): For each value of $\mathrm{Da}$, the reaction efficiency computed according to numerical differentiation of the data in (a) is shown by markers, and the theoretical asymptotic value $\varepsilon_{\infty}$, computed based on the first and second moments of the same first passage and return data, is shown as a dashed line.

Eq. (47) with $\rho=2$, whereas for higher Da deviations from the well-mixed behavior are observed because transverse diffusion is incapable of homogenizing the cross-section under fast reaction. For $\mathrm{Da}=10^{2}$, the numerical simulations agree with the fully-transport-limited solution $M(t)=M_{0} \Psi_{0}(t)$.

The reaction efficiency $\varepsilon(t)$ corresponding to these dynamics is shown in Fig. 5b. The theoretical asymptotic efficiency $\varepsilon_{\infty}$, Eq. (55), evaluated using the first and second moments of the numerically-determined first passage and return times, is in good agreement with the simulations. Although some quantitative differences are discernible in the mass decay, compare Figures $5 \mathrm{a}$ and $2 \mathrm{a}$, the reaction dynamics are remarkably similar to the two-dimensional channel case, especially regarding the reaction efficiency, compare Figures 5b and $3 \mathrm{a}$.

\subsubsection{Reactive transport in a body centered cubic beadpack}

We now investigate the application of our approach to the case of reactive transport in a crystalline porous medium. Recall that the inter-reaction time 
approach presented here relies on the assumption that a statistical description of the return times, with stochastic return times but with the same statistics applying to each return to the interface, is sufficient to characterize reaction. We assess this hypothesis for advective-diffusive transport in a body centered cubic beadpack [75], where the structure of the medium is periodic but not all points on the interface within a unit cell are equivalent in their positioning relative to the rest of the interface (Fig. 1b, (iii)). For details on the numerical simulations, see Appendix A. As shown in Appendix F, the interface-extent coefficient for this system is given by

$$
\rho=\frac{3 \pi}{2}\left(1-\frac{\sqrt{3} \pi}{8}\right)^{-1} \approx 14.7 .
$$

The relative importance of advection compared to diffusion can be quantified through the Péclet number, which we define in terms of the absolute value $\bar{v}$ of the Eulerian mean velocity vector and the characteristic lengthscale $\ell_{c}$ as

$$
\widehat{P} \mathrm{e}=\frac{\ell_{c} \bar{v}}{D} .
$$

The total mass as a function of time for different Damköhler numbers and $\mathrm{Pe}=10^{3}$ is shown in Fig. 6a, for an initial condition uniformly distributed over a conventional unit cell (see Fig. 1b, (iii)). The inter-reaction time approach, parameterized according to the numerically-determined first passage and return tails $\Psi_{0}$ and $\Psi_{d}$ as discussed above, is in good agreement with the reactive PTRW simulations. For low Da, the mass evolution agrees with the effective well-mixed decay, Eq. (46). This happens because, for low reaction rates, the fluid reactant remains homogeneous over a representative region of pore volume. As for the channel examples, deviations from the well-mixed behavior become more pronounced as the Damköhler number increases and transport is unable to efficiently homogenize the fluid-phase concentration. The corresponding reaction efficiency $\varepsilon(t)$ is shown in Fig. 5b. The theoretical asymptotic efficiency $\varepsilon_{\infty}$, Eq. (55), evaluated using the first and second moments of the numerically-determined first passage and return times, is again in good agreement with the simulations. In this case, the homogeneous character of the initial condition results in a reaction efficiency that is initially unity. For high Damköhler, reaction tends to destroy homogeneity, which results in a decrease of the reaction efficiency towards the asymptotic value. 


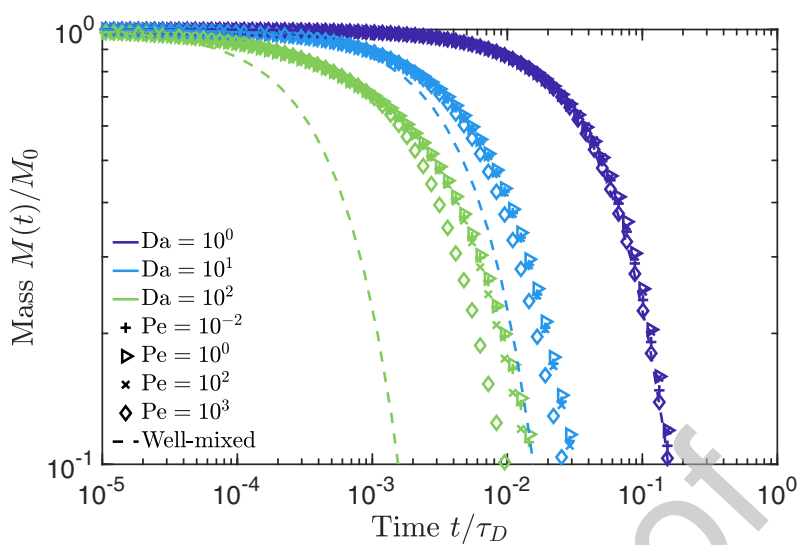

Figure 7: Total mass as a function of time in a body centered cubic beadpack, for different Damköhler numbers Da (colors) and Péclet numbers Pe (symbols). The initial condition is homogeneous in a conventional unit cell. Results are computed from numerical inversion of Eq. (30) given numerical computation of the first passage and return times. Dashed lines correspond to the analytical solution with the effective well-mixed reaction rate (45). First passage statistics are computed from $10^{4}$ samples and return statistics from $10^{5}$ samples, both using a time step $\Delta t=10^{-7} \tau_{D}$.
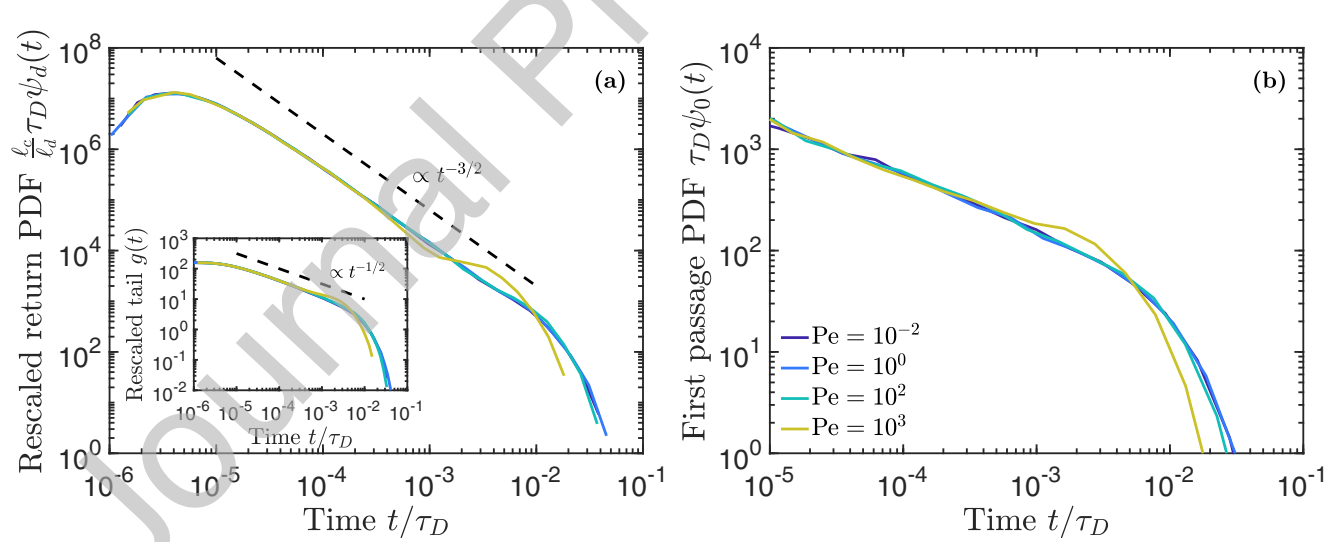

Figure 8: Rescaled first return time PDF (a) and first passage time PDF (b) for a homogeneous initial condition in a conventional unit cell for advective-diffusive transport in a body centered cubic beadpack for different Péclet numbers (colors). Return time statistics are computed from $10^{5}$ return time samples and first passage time statistics from $10^{4}$ samples, both using a time step $\Delta t=10^{-7} \tau_{D}$. The inset shows the rescaled return time tail probability, computed from the same data. The large-time scalings associated with pure diffusion in a semi-infinite domain, which here hold below the characteristic time to encounter a different bead and above the discretization timescale $\tau_{d}=\ell_{d}^{2} /(2 D)=\tau_{D} \ell_{d}^{2} / \ell_{c}^{2}$, are shown as dashed lines. 
In Fig. 7, we show the total mass as a function of time for the same Damköhler numbers as in Fig. 6 and different values of Péclet number, computed using the inter-reaction time approach. Although we refrain from showing these results in Fig. 6 to avoid clutter, we verified that PTRW simulations show similar very good agreement as for Fig. 6a across Péclet numbers. For sufficiently high Da, the reaction is slower than the effective well-mixed prediction, but approaches the latter when the Péclet number becomes sufficiently high, as advection-induced transverse dispersion effects become important compared to diffusive mixing. In order to better understand the role of the Péclet number in the reaction dynamics, we examine the first passage and return time statistics arising under different transport regimes, see Fig. 8. The flow considered here is known to induce chaotic mixing [75, 77-79]. This means that the flow efficiently homogenizes the concentration on the cross-section of the pore space transverse to the mean flow direction, over a characteristic advective mixing timescale. For sufficiently low Péclet number, as long as the advective mixing timescale is large compared to the typical diffusive first passage and return times, transverse mixing is carried out by diffusion and adyection does not have an appreciable effect on reaction. Thus, the first passage and return times, along with the mass dynamics, are similar for Pe up to $10^{2}$. When advective effects become important, particles far from the interface are brought towards it faster than by diffusion alone. On the other hand, particles at intermediate distances can take longer to reach the interface than they would have by diffusion. Lastly, very low return times remain controlled by diffusion. This effect on the first passage and return times can be clearly seen for $\mathrm{Pe}=10^{3}$.

Whichever effect is dominant, the average return time reflects the fact that at sufficiently late times concentration is well mixed, and remains given by Eq. (50). Thus, the low-Da reaction behavior, which depends only on the average return time, is unaffected by the mixing mechanism and remains unchanged, see Fig. 7. However, the distributions of first passage and return times become less broad with increasing Péclet (Fig. 8), and the effective well-mixed regime is reached faster. For this reason, the reaction dynamics approach the effective well-mixed behavior at higher values of the Damköhler number; the strength of this effect increases with increasing Pe (Fig. 7). We note that for higher Péclet numbers, Pe $\gtrsim 10^{4}$, the corresponding stronglyadvection-dominated transport simulations become particularly sensitive to the underlying flow velocities. Accurate results for such cases would require more finely-resolved flow fields, and we refrain from simulating them here. 
The first passage and return time dynamics in the two- and three-dimensional channel and beadpack examples exhibit some qualitative similarities, due to the role of diffusion in transverse mixing and its dominant role near the fluid-solid interface. Indeed, the corresponding distributions exhibit clear diffusive scalings, followed for long times by cutoffs, see Figs. 4 and 8. The characteristic timescale associated with the cutoff, and therefore the characteristic variability in inter-reaction times, depends on medium geometry, and in the case of the beadpack is also impacted by advective mixing for large Péclet number. Correspondingly, the reactant mass dynamics in these different systems exhibit similarities, such as late-time exponential decay, but also differences in the onset of transport limitations with Damköhler number and the effective reaction rates associated with a well-mixed system, see Figs. 2, 5,6 , and 7 . In particular, the interface extent coefficient $\rho$, quantifying the available surface area per unit volume, increases compared to the $2 \mathrm{~d}$ channel by a factor of 2 in the $3 \mathrm{~d}$ channel and about 15 in the beadpack, effectively increasing the well-mixed reaction rate in these systems for a given value of the Damköhler number. Transport limitation effects become important for $\rho \mathrm{Da} \gtrsim 1$, although they are mitigated in the beadpack when the Péclet number is large and advection-induced mixing plays a role.

\section{Discussion and conclusions}

In this work, we have developed a new framework to quantify the effect of transport limitations on fluid-solid reaction dynamics in porous media. Our approach, based on the chemical continuous time random walk theory of inter-reaction times, relates the statistics of solute excursions away from and back to the fluid-solid interface to reaction times. We have shown that the dynamics of effective reaction rates relate to the statistics of inter-reaction times, which are in turn controlled by transport and medium geometry. We have illustrated the approach analytically for advection-diffusion-reaction in stratified flow through a two-dimensional channel, and provided a generic numerical approach to determine the corresponding dynamics in more complex media and flow fields. For fast reactions, reactant mass is controlled by the first passage time of solute to the reactive solid interface. For slow reactions, multiple excursions to the interface are necessary before reaction occurs. In the latter case, the statistics of the durations of these excursions, which are sensitive to diffusive mass transfer near the interface, become a dominant control on mass dynamics. Consistently with numerical simulations, our theory 
predicts that, for intermediate and high Damköhler number, these effects can lead to significant reaction slowdown due to transport limitations, even for the simple reaction studied here, and even in simple geometries. We provide analytical expressions for the late-time effective reaction rate as a function of Damköhler number, which exhibits a transition from the well-mixed reaction rate at low Da to the inverse of the diffusion time at large Da, a direct consequence of transport limitations.

The theory presented here also leads to a useful numerical framework. Fluid-solid reactions pose unique computational and theoretical challenges, as they require resolving transport dynamics close to the interface in a consistent manner. Here, we have analyzed a simple fluid solid reaction in detail, and shown how the assumption of scale separation between reaction and transport dynamics near the interface leads to a consistent continuum model of surface reaction. Resolved numerical simulations of these dynamics are computationally demanding. The inter-reaction time approach presented here opens up new possibilities for efficient numerical computation of mass dynamics by extending existing theoretical frameworks for the determination of first passage times [34, 47, 55, 80] and/or numerical techniques for efficient first passage time computation [81-83].

This work has been mainly concerned with introducing the concepts and methodology underlying a new approach to upscaling fluid-solid reaction dynamics. As such, we have made significant simplifications regarding the reaction chemistry and porous medium structure. The framework developed here brings new perspectives to link the statistical characteristics of medium geometry and flow to reaction dynamics in a broad range of porous media, which will be the subject of future work. Furthermore, we expect an approach similar to that presented here to be applicable to the dynamics of mass breakthrough as a function of distance, in terms of the statistics of interreaction distances. Future work will also aim to generalize the approach to higher-order reactions involving multiple transported components, multiple simultaneous reactions, and heterogeneity (spatial and temporal variability) in the solid-phase reactant distribution along the interface.

\section{Acknowledgments}

TA is supported by a Marie Skłodowska Curie Individual Fellowship, under the project ChemicalWalks 792041. TLB gratefully acknowledges funding by the ERC under the project ReactiveFronts 648377 . The authors thank 
Joris Heyman for providing the numerical flow data used in the beadpack PTRW simulations and Guillem Sole-Mari for useful comments and suggestions. A $\mathrm{C}++$ implementation of the particle tracking algorithms used to generate the numerical data employed in this work are available under an open-source license (github: github.com/tcAquino/BeadPack, DOI: 10.5281/zenodo.4392882). Please contact the authors for further information or requests.

\section{Appendix A. Particle tracking simulations}

In this appendix, we discuss some details of the PTRW (particle tracking random walk) simulation method used in this work. Lagrangian particle tracking methods, which simulate particle trajectories based on a random walk, have been found to mitigate numerical dispersion issues, typically associated with more traditional Eulerian methods, and their impact on fluidfluid reactions [84]. However, particle tracking methods are less developed and widespread than Eulerian methods, and we are not aware of an available tool for Lagrangian simulation of fluid-solid reaction dynamics. In the present work, a PTRW approach has the added benefit of directly simulating Lagrangian trajectories, the properties of which are the basis for the theoretical developments. This allows us to employ the same methodology to simulate the conservative component of the reactive transport problem and to determine first passage and return times (see Sections 5 and 6 of the main text).

The PTRW method used here is based on that employed in [85] to simulate conservative transport in a number of stratified flows, where it was validated against theoretical predictions for dispersion, concentration distributions, breakthrough curves (first passage times across a plane), and Lagrangian velocity distributions. The conservative transport algorithm consists in discretizing the Langevin equation (1) for a set of Lagrangian particles, or trajectories, with prescribed initial conditions. The displacement $\Delta \boldsymbol{X}(t)$ of a particle in a time step of duration $\Delta t$ starting at time $t$ is computed as the sum of the advective and diffusive contributions to the change in particle position $\boldsymbol{X}(t)$,

$$
\Delta \boldsymbol{X}(t)=\boldsymbol{X}(t+\Delta t)-\boldsymbol{X}(t)=\Delta \boldsymbol{X}_{A}(t)+\Delta \boldsymbol{X}_{D}(t) .
$$

Boundary conditions at the solid interface are implemented as elastic collisions based on the full displacement $\Delta \boldsymbol{X}(t)$. The diffusive contribution is 
obtained from a stochastic forward-Euler scheme as $\Delta \boldsymbol{X}_{D}(t)=\sqrt{2 D \Delta t} \boldsymbol{\xi}(t)$, where the component of $\boldsymbol{\xi}(t)$ along each Cartesian axis and at each time $t$ is sampled independently from a unit (zero mean and unit variance) Gaussian distribution. For the two-dimensional channel simulations of Section 5, the advective contribution is also computed based on a forward-Euler scheme, $\Delta \boldsymbol{X}_{A}(t)=\boldsymbol{v}[\boldsymbol{X}(t)]$, where the velocity field $\boldsymbol{v}$ is evaluated at particle positions according to the analytical flow field, Eq. (35).

Here, we have extended the algorithm to account for: (i) elastic reflections on bead surfaces, representing reflecting boundary conditions; (ii) advection due to an arbitrary velocity field, previously obtained and provided at a number of points on an arbitrary (possibly unstructured) grid; and (iii) fluid-solid reaction. Regarding (i), we implemented and independently verified a simple trigonometry-based computation of perfectly elastic collisions on spherical surfaces, valid in arbitrary dimension (for circles in the plane, etc.). Regarding (ii), the flow field at a given position is obtained from linear interpolation based on Delaunay triangulation and Sibson natural neighbor coordinates on an arbitrary grid, based on the well-established CGAL C++ library [86]. For the beadpack simulations, the advective particle displacement associated with the interpolated flow is computed according to a fourth-order explicit Runge-Kutta scheme. Finally, (iii) is implemented according to the considerations of Section 2. As discussed therein, the spatial support scale $\ell_{d}$ should be large compared to $\sqrt{2 D \Delta t}$, in order to permit resolving fluid-reactant concentrations near the interface and the resulting reaction rates. We set the support scale

$$
\ell_{d}=10 \sqrt{2 D \Delta t}
$$

It is important to note that $\ell_{d}$ is not a parameter of the theoretical model, but is rather associated with the discretization. In this sense, taking $\ell_{d}=$ $a \sqrt{2 D \Delta}$ with $a>10$ leads to the same results in the continuum limit $\Delta t \rightarrow$ 0 . It is, however, necessary to take $a \gtrsim 10$, in order to guarantee that reactant concentrations near the interface are resolved correctly and converge for $\Delta t \rightarrow 0$. The diffusion time associated with the support scale $\ell_{d}$ is $\tau_{d}=$ $\ell_{d}^{2} / 2 D$; thus, our choice corresponds for a given $\ell_{d}$ to setting $\Delta t=\tau_{d} / 100$.

The algorithm employed to compute first passage and return times is validated here by the agreement with theoretical results for the one-dimensional channel, see Section 5. Similarly, the agreement between theory and simulations found for the dynamics of total mass, especially regarding the theoretical results for low and high Damköhler number and the assymptotic reaction 
rates for arbitrary Damköhler number, discussed in Sections 5 and 6, provides validation for the reactive transport algorithm. We note that we have implemented only the $A_{F}+B_{S} \rightarrow B_{S}$ reaction studied here, but the reaction algorithm can be direclty extended to other reactions and heterogeneous distributions of solid-phase reactants at the expense of explicitly keeping track of the surface concentrations of the latter across the fluid-solid interface. A github repository where the code is available under an open-source license and regularly updated can be found at github.com/tcAquino/BeadPack. Further benchmarks and extensions to the fluid--solid reaction algorithm will be included and discussed thereat. The version of the code used in the present work can be found at the DOI 10.5281/zenodo.4392882. Additional documentation addressing further technical details may also be found in both these repositories.

In order to simulate reactive transport and determine first passage and return times using the PTRW algorithm, we first require the flow field, which may be given at a set of points on an arbitrary grid. For the beadpack simulations of Section 6, the flow field was obtained numerically from Stokes flow simulations using a finite volume discretization in openFOAM [87], with $N_{c}=1572138$ hexahedral cells. The flow was computed in a conventional (cubic) unit cell, of side $\ell_{c}=1 \mathrm{~cm}$, of the body centered cubic packing considered. The associated body cubic centered bead diameter is $d=\sqrt{3} \ell_{c} / 2$ [76], corresponding to a spatial resolution of about $\ell_{c} / N_{c}^{1 / 3} \approx 10^{-2} d$. Periodic boundary conditions were imposed on the cell faces and no-slip boundaries at the spherical bead interfaces. The flow had kinematic viscosity $5 \cdot 10^{-7} \mathrm{~m}^{2} / \mathrm{s}$ and was driven by a pressure gradient of $1 \mathrm{~Pa} / \mathrm{m}$, obtained by applying a body force. In terms of Cartesian axes perpendicular to the unit cell faces, the mean velocity vector had an orientation corresponding to an angle $5 \pi / 40$ with the $x$ axis on the $x-y$ plane and an angle $\pi / 40$ with the $z$ axis. This flow configuration has been analyzed in [75], and it is known to induce chaotic mixing. As discussed in the same reference, the contact points between beads can cause numerical instabilities in the numerical determination of the flow field. This issue can be addressed by placing small spheres or cylinders, with no-slip boundary conditions on the exposed surface, at the contact points between beads to avoid numerical issues near the contact points. These structures do not have an appreciable impact on flow and transport properties [75]. In the present simulations, spheres of $20 \%$ the bead diameter were employed in this manner. These contact spheres are not needed for sta- 


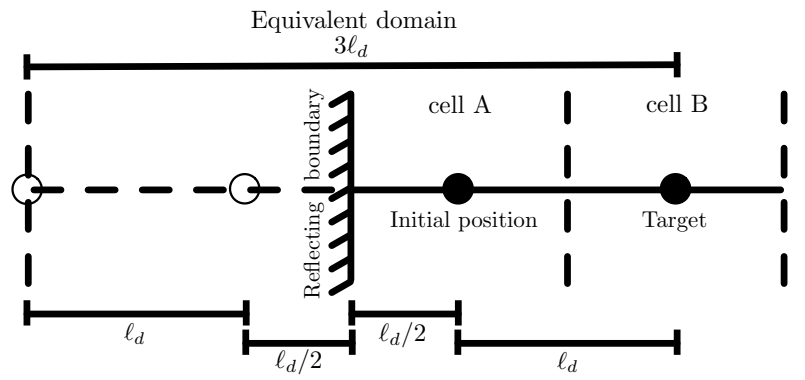

Figure B.9: Schematic illustrating the equivalence of the first passage time by diffusion in one dimension from the center of cell $A$ to the center of cell $B$, in the presence of a reflecting boundary and in an equivalent bounded domain. If the boundary, at distance $\ell_{d} / 2$ from the starting point, is reached before the center of cell $B$, at a distance $\ell_{d}$, the first passage time to the latter is the same as to its reflection. Thus, the first passage time in the presence of the boundary is the same as the first passage time to either boundary in the equivalent domain of length $3 \ell_{d}$.

bility of the PTRW simulations; in the conservative and reactive transport simulations, no boundary conditions were enforced on their surface, and zerovelocity points were added at the contact points instead. Particle positions undergoing advection-diffusion were mapped onto the unit cell according to the periodic boundary conditions.

\section{Appendix B. Statistics of single interface visit times}

Here, we obtain the $\mathrm{PDF}$ of the time spent in the reactive region in each visit. Consider again one-dimensional diffusion close to the interface. The first passage time from the middle of the discretization cell nearest the interface to the middle of the adjacent cell corresponds to the duration of a visit to the reactive region. It can be obtained by considering the first passage time to either boundary in a domain of length $3 \ell_{d}$ and starting from a distance $\ell_{d}$ from one boundary, as illustrated in Fig. B.9. The first passage time PDF to reach either boundary in a domain of length $L$, starting from a distance $\ell$ from one and $L-\ell$ from the other, has Laplace transform [71]

$$
\tilde{\psi}(\lambda ; \ell, L)=\frac{\operatorname{csch}\left[\sqrt{\ell^{2} \lambda / D}\right]+\operatorname{csch}\left[\sqrt{(L-\ell)^{2} \lambda / D}\right]}{\operatorname{coth}\left[\sqrt{\ell^{2} \lambda / D}\right]+\operatorname{coth}\left[\sqrt{(L-\ell)^{2} \lambda / D}\right]} .
$$

Setting $L=3 \ell_{d}$ and $\ell=\ell_{d}$ yields the single-visit PDF

$$
\tilde{\psi}_{v}(\lambda)=\left[2 \cosh \left(\sqrt{2 \tau_{d} \lambda}\right)-1\right]^{-1} .
$$


Expanding for $\lambda \ll 1 / \tau_{d}$ and inverting the resulting Laplace transform leads to Eq. (19). Recall that $\lambda \ll 1 / \tau_{d}$ represents large times compared to the discretization time, so that this result constitutes a step in taking the continuum limit rather than a large-time approximation. As before, the same conclusion is reached if we consider the first passage times to within $\ell_{d}$ of the interface starting from a distance $2 \ell_{d}$ from the latter. This result remains valid in higher dimensions under the assumption that interface is locally flat at the scale of the transport model.

\section{Appendix C. Impact of first passage time to the interface on reac- tion dynamics}

According to the chCTRW framework, in the absence of the delay associated with first reaching the interface, the generalized rate law governing the evolution of total fluid reactant mass is given by Eq. (8). When this delay is added, the mass is equal to the initial mass for times smaller than the delay, and evolves according to the previous equation for later times. That is, averaging over initial delays (i.e., over an ensemble of Lagrangian particles distributed according to the initial condition),

$$
M(t)=M_{0} \int_{0}^{t} d t^{\prime} \psi_{0}\left(t^{\prime}\right)+\int_{0}^{t} d t^{\prime} \psi_{0}\left(t^{\prime}\right) M_{\phi}\left(t-t^{\prime}\right) .
$$

The first term represents the fact that, if the interface has not been reached for the first time by time $t$, which happens with probability $\int_{0}^{t} d t^{\prime} \psi_{0}\left(t^{\prime}\right)$ over the ensemble of Lagrangian particles, the fluid mass is equal to its initial value $M(0)=M_{0}$. Regarding the second term, if the interface is reached for the first time at time $t^{\prime}<t$, which happens with probability density $\psi_{0}\left(t^{\prime}\right)$, the mass at time $t$ is equal to $M_{\phi}\left(t-t^{\prime}\right)$. Note that, given a first arrival at the interface at time $t=t^{\prime}, M\left(t=t^{\prime}\right)=M_{\phi}(0)=M_{0}$. Then, for times $t>t^{\prime}$, the mass dynamics proceed according to the inter-reaction times, with $M_{\phi}$ obeying the chCTRW equation (8). This result may be written in terms of the first passage time tail probability $\Psi_{0}$ as

$$
M=M_{0} \Psi_{0}+M_{\phi} * \psi_{0} .
$$

Substituting Eq. (8) for the dynamics of mass $M_{\phi}$ resulting from the interreaction times, we obtain Eq. (25). 


\section{Appendix D. Mass dynamics and reactive time}

The dynamics of reactant mass may be formulated in terms of the statistics of the total time spent in the reactive region rather than the statistics of the inter-reaction times. In this appendix, we show that the former approach leads to the same results as the latter, which is developed in the main text. Consider the reactive time $U_{d}(t)$, representing the total time spent within distance $\ell_{d}$ of the interface. We denote its $\operatorname{PDF}$ by $p_{U}(\cdot ; t)$, that is, $p_{U}(u ; t) d u$ is the probability that, given total elapsed time $t$, a particle has spent a time in the interval $[u, u+d u)$ within distance $\ell_{d}$ of the reactive interface.

For small $\ell_{d}$, in preparation for taking the continuum limit $\ell_{d} \rightarrow 0$, we approximate

$$
U_{d}(t) \approx 2 \tau_{d} N_{d}(t)
$$

where $N_{d}(t)$ is the (random) number of visits to the reactive region by time $t$, each of which contributes the mean residence time per visit $2 \tau_{d}$, see Eq. (19). Since $U_{d}(t)$ is proportional to $N_{d}(t)$, denoting by $p_{N}(n ; t)$ the probability that $N_{d}(t)=n$, we have

$$
p_{U}(u ; t) \approx p_{N}(0 ; t) \delta(u)+\left(2 \tau_{d}\right)^{-1} p_{N}\left(\frac{u}{2 \tau_{d}} ; t\right)
$$

where $\delta(\cdot)$ is the Dirac delta.

The number of visits to the reactive region by a given time depends on the PDF of inter-visit delay times $\psi_{d}$, as well as on the PDF $\psi_{0}$ of the time of the first visit. Adapting the results of [72], the Laplace transform of the distribution of the number of visits to the reactive region with respect to time $t$ in terms of these quantities is given by

$$
\tilde{p}_{N}(n ; \lambda)=\left\{\begin{array}{ll}
\tilde{\Psi}_{0}(\lambda), & n=0 \\
\tilde{\Psi}_{d}(\lambda) \tilde{\psi}_{0}(\lambda) \tilde{\psi}_{d}(\lambda)^{n-1}, & n>0
\end{array} .\right.
$$

In the limit of small $\ell_{d}$, we obtain, according to Eq. (D.2),

$$
\tilde{p}_{U}(u ; \lambda)=\tilde{\Psi}_{0} \delta(u)+\lambda^{-1} \tilde{\psi}_{0}(\lambda) E_{d}(u, \lambda),
$$

where

$$
E_{d}(u, \lambda)=\frac{\lambda \tilde{g}(\lambda) \ell_{c}}{\ell_{d}} \exp \left[-\frac{\lambda \tilde{g}(\lambda) \ell_{c}}{\ell_{d}} u\right]
$$


with the rescaled tail probability $g$ defined in terms of the return time tail $\Psi_{d}$ according to Eq. (16).

To quantify the dynamics of total mass in terms of the reactive time $U_{d}(t)$, we make use of a subordination-type description [31, 69, 73, 74]. Within the reactive region, reaction is well-mixed in the sense described in Section 2. The overall reaction then proceeds according to the time particles spend within this region. The amount of mass left by time $t$ is the average of the surviving mass $M_{0} \exp \left[-k_{d} U_{d}(t)\right]$ over all possible times $U_{d}(t)$ spent within $\ell_{d}$ of the interface up to time $t$, which are distributed according to $p_{U}(\cdot ; t)$. That is,

$$
M(t)=M_{0} \int_{0}^{\infty} d u e^{-k_{d} u} p_{U}(u ; t) .
$$

The Laplace transform of the total mass (26) obtained using the interreaction time formulation is then recovered by direct computation in the limit $\ell_{d} \rightarrow 0$.

In terms of a Laplace transform with respect to operational time (denoted by a hat), keeping time $t$ fixed, we may write Eq. (D.6) as

$$
\frac{M(t)}{M_{0}}=\hat{p}_{U}\left(k_{d} ; t\right),
$$

which can also be interpreted as the Laplace transform of the stochastic process $U_{d} / \ell_{d}$, evaluated at the rate $\ell_{d} k_{d}=k c_{A}$. In the limit $\ell_{d} \rightarrow 0$ of fine discretization, the stochastic process $U_{d} / \ell_{d}$ is the local time at the boundary mentioned in the introduction [38-41] (note that, in accordance with the standard terminology used in the literature, the so-called local time has units of time per length). It is this quantity, rather than $U_{d}$ itself, that is well defined in the continuum limit. This is directly related to the fact that a particle undergoing continuous diffusion in one dimension returns to the initial position infinitely many times within an arbitrarily small time interval. Despite the fact that this represents a mathematical abstraction, it corresponds to the correct behavior when transport is adequately described by continuous diffusion at the scale of interest.

\section{Appendix E. First passage times to the wall in a 2D channel}

In this appendix, we provide the Laplace transforms of the first passage time PDFs of solute to the channel walls for transport in stratified flow 
through a two-dimensional straight channel. As discussed in Section 5, these are controlled by diffusion in the transverse direction, although the velocity profile can impact the result due to its role in determining the initial distribution, for example for flux-weighted injections.

The different first passage times are obtained by using Eq. (27) for a point injection, and weighting according each initial condition. Thus, the mid-channel injection (a) leads to

$$
\tilde{\psi}_{0}(\lambda)=\tilde{\psi}\left(\lambda ; \ell_{c}, 2 \ell_{c}\right)=\operatorname{sech}\left(\sqrt{2 \tau_{D} \lambda}\right) .
$$

For the homogeneous injection (b), we have

$$
\tilde{\psi}_{0}(\lambda)=\frac{1}{2 \ell_{c}} \int_{0}^{2 \ell_{c}} d \ell \tilde{\psi}\left(\lambda ; \ell, 2 \ell_{c}\right)=\frac{\tanh \left(\sqrt{2 \tau_{D} \lambda}\right)}{\sqrt{2 \tau_{D} \lambda}} .
$$

For the flux-weighted case (c), we find

$$
\tilde{\psi}_{0}(\lambda)=\frac{1}{2 \ell_{c}} \int_{0}^{2 \ell_{c}} d \ell \frac{v\left(\ell-\ell_{c}\right)}{\bar{v}} \tilde{\psi}\left(\lambda ; \ell, 2 \ell_{c}\right)=\frac{3}{2 \tau_{D} \lambda}\left[1-\frac{\tanh \left(\sqrt{2 \tau_{D} \lambda}\right)}{\sqrt{2 \tau_{D} \lambda}}\right],
$$

and for all mass starting at the channel walls (d),

$$
\tilde{\psi}_{0}(\lambda)=1 \text {. }
$$

\section{Appendix F. Interface-extent coefficient for the body centered cu- bic beadpack}

In order to compute the interface-extent coefficient $\rho$ for a body centered cubic beadpack, first note that the bead radius is related to the conventional (cubic) unit cell side by $r=\sqrt{3} \ell_{c} / 4$, where we have taken the cell side $\ell_{c}$ as the characteristic length (see, e.g., [76] on the theory of crystalline structures). Within a unit cell, there is a full bead at the center, and eight bead quarters at each cell corner, totaling a solid volume of two full beads. Since the volume of a bead is $V_{b}=4 \pi r^{3} / 3$, the porosity is given by

$$
\varphi=1-\frac{2 V_{b}}{\ell_{c}^{3}}=1-\frac{\sqrt{3} \pi}{8} \approx 0.320 .
$$


The surface area of a bead is $A_{b}=4 \pi r^{2}$, so that, according to Eq. (48), we have

$$
\rho=\frac{2 A_{b} \ell_{c}}{\varphi \ell_{c}^{3}}=\frac{3 \pi}{2}\left(1-\frac{\sqrt{3} \pi}{8}\right)^{-1} .
$$

\section{References}

[1] C. I. Steefel, D. J. DePaolo, P. C. Lichtner, Reactive transport modeling: An essential tool and a new research approach for the earth sciences, Earth Planet. Sci. Lett 240 (3-4) (2005) 539-558.

[2] L. Li, K. Maher, A. Navarre-Sitchler, J. Druhan, C. Meile, C. Lawrence, J. Moore, J. Perdrial, P. Sullivan, A. e. a. Thompson, Expanding the role of reactive transport models in critical zone processes, Earth-science reviews 165 (2017) 280-301.

[3] K. Maher, A. Navarre-Sitchler, Reactive transport processes that drive chemical weathering: From making space for water to dismantling continents, Rev. Mineral. Geochem. 85 (1) (2019) 349-380.

[4] K. Maher, K. U. Mayer, The art of reactive transport model building, Elements 15 (2) (2019) 117-118.

[5] F. Chapelle, Ground-Water Microbiology and Geochemistry, John Wiley and Sons, 2001.

[6] C. A. J. Appelo, D. Postma, Geochemistry, groundwater and pollution, CRC press, 2004.

[7] S. L. Brantley, J. D. Kubicki, A. F. White, Kinetics of water-rock interaction, Vol. 168, Springer, 2008.

[8] S. Molins, D. Trebotich, C. I. Steefel, C. Shen, An investigation of the effect of pore scale flow on average geochemical reaction rates using direct numerical simulation, Water Resour. Res. 48 (3) (2012).

[9] S. Molins, D. Trebotich, L. Yang, J. B. Ajo-Franklin, T. J. Ligocki, C. Shen, C. I. Steefel, Pore-scale controls on calcite dissolution rates from flow-through laboratory and numerical experiments, Environ. Sci. Technol. 48 (13) (2014) 7453-7460. 
[10] L. E. Beckingham, E. H. Mitnick, C. I. Steefel, S. Zhang, M. Voltolini, A. M. Swift, L. Yang, D. R. Cole, J. M. Sheets, J. B. Ajo-Franklin, et al., Evaluation of mineral reactive surface area estimates for prediction of reactivity of a multi-mineral sediment, Geochim. Cosmochim. Acta 188 (2016) 310-329.

[11] C. Soulaine, S. Roman, A. Kovscek, H. A. Tchelepi, Mineral dissolution and wormholing from a pore-scale perspective, J. Fluid Mech. 827 (2017) 457-483.

[12] L. E. Beckingham, C. I. Steefel, A. M. Swift, M. Voltolini, L. Yang, L. M. Anovitz, J. M. Sheets, D. R. Cole, T. J. Kneafsey, E. H. Mitnick, et al., Evaluation of accessible mineral surface areas for improved prediction of mineral reaction rates in porous media, Geochim. Cosmochim. Acta 205 (2017) 31-49.

[13] H. Deng, S. Molins, D. Trebotich, C. Steefel, D. DePaolo, Pore-scale numerical investigation of the impacts of surface roughness: Upscaling of reaction rates in rough fractures, Geochim. Cosmochim. Acta 239 (2018) 374-389.

[14] I. Battiato, D. Tartakovsky, Applicability regimes for macroscopic models of reactive transport in porous media, J. Contam. Hydrol. 120 (2011) $18-26$.

[15] I. Battiato, D. M. Tartakovsky, A. M. Tartakovsky, T. Scheibe, On breakdown of macroscopic models of mixing-controlled heterogeneous reactions in porous media, Adv. Water Resour. 32 (11) (2009) 16641673.

[16] J-M. Vanson, F.-X. Coudert, B. Rotenberg, M. Levesque, C. Tardivat, M. Klotz, A. Boutin, Unexpected coupling between flow and adsorption in porous media, Soft matter 11 (30) (2015) 6125-6133.

[17] J.-M. Vanson, F.-X. Coudert, M. Klotz, A. Boutin, Kinetic accessibility of porous material adsorption sites studied through the lattice boltzmann method, Langmuir 33 (6) (2017) 1405-1411.

[18] J.-M. Vanson, A. Boutin, M. Klotz, F.-X. Coudert, Transport and adsorption under liquid flow: the role of pore geometry, Soft Matter 13 (4) (2017) 875-885. 
[19] T. Sherman, A. Paster, G. Porta, D. Bolster, A spatial Markov model for upscaling transport of adsorbing-desorbing solutes, J. Contam. Hydrol. 222 (2019) 31-40.

[20] N. Sund, D. Bolster, C. Dawson, Upscaling transport of a reacting solute through a peridocially converging-diverging channel at pre-asymptotic times, J. Contam. Hydrol. 182 (2015) 1-15.

[21] L. Li, C. Peters, M. Celia, Effects of mineral spatial distribution on reaction rates in porous media, Water Resour. Res. 43 (1) (2007) W01419.

[22] L. Li, C. I. Steefel, L. Yang, Scale dependence of mineral dissolution rates within single pores and fractures, Geochim. Cosmochim. Acta 72 (2) (2008) 360-377.

[23] C. Soulaine, H. A. Tchelepi, Micro-continuum approach for pore-scale simulation of subsurface processes, Transp. Porous Media 113 (3) (2016) 431-456.

[24] P. K. Kang, E. Bresciani, S. An, S. Lee, Potential impact of pore-scale incomplete mixing on biodegradation in aquifers: From batch experiment to field-scale modeling, Adv. Water Resour. 123 (2019) 1-11.

[25] M. Dentz, T. Le Borgne, A. Englert, B. Bijeljic, Mixing, spreading and reaction in heterogeneous media: a brief review, J. Contam. Hydrol. 120 (2011) 1-17.

[26] M. Dentz, P. Gouze, J. Carrera, Effective non-local reaction kinetics for transport in physically and chemically heterogeneous media, J. Contam. Hydrol. 120 (2011) 222-236.

[27] Z. Alhashmi, M. Blunt, B. Bijeljic, Predictions of dynamic changes in reaction rates as a consequence of incomplete mixing using pore scale reactive transport modeling on images of porous media, J. Contam. Hydrol. 179 (2015) 171-181.

[28] D. T. Gillespie, Exact stochastic simulation of coupled chemical reactions, J. Phys. Chem. 81 (25) (1977) 2340-2361.

[29] D. T. Gillespie, A rigorous derivation of the chemical master equation, Physica A 188 (1-3) (1992) 404-425. 
[30] M. von Smoluchowski, Mathematical theory of the kinetics of the coagulation of colloidal solutions, Z. phys. Chem. 92 (1917) 129-168.

[31] T. Aquino, M. Dentz, Chemical continuous time random walks, Phys. Rev. Lett. 119 (23) (2017) 230601.

[32] B. Berkowitz, A. Cortis, M. Dentz, H. Scher, Modeling non-Fickian transport in geological formations as a continuous time random walk, Rev. Geophys. 44 (2) (2006).

[33] H. H. McAdams, A. Arkin, Stochastic mechanisms in gene expression, Proc. Natl. Acad. Sci. U.S.A. 94 (3) (1997) 814-819.

[34] O. Bénichou, C. Chevalier, J. Klafter, B. Meyer, R. Voituriez, Geometrycontrolled kinetics, Nature Chemistry 2 (6) (2010) 472-477.

[35] O. Bénichou, D. Grebenkov, P. Levitz, C. Loverdo, R. Voituriez, Optimal reaction time for surface-mediated diffusion, Phys. Rev. Lett. 105 (15) (2010) 150606.

[36] Y. Meroz, I. M. Sokoloy, J. Klafter, Distribution of first-passage times to specific targets on compactly explored fractal structures, Phys. Rev. E 83 (2) (2011) 020104(R).

[37] A. Godec, R. Metzler, Universal proximity effect in target search kinetics in the few-encounter limit, Phys. Rev. X 6 (4) (2016) 041037.

[38] I. S. Karatzas, S. Shreve, Brownian motion and stochastic calculus, Vol. 113, Springer, 1988.

[39] L. Takács, On the local time of the brownian motion, Ann. Appl. Probab. (1995) 741-756.

[40] D. Grebenkov, Residence times and other functionals of reflected Brownian motion, Phys. Rev. E 76 (4) (2007) 041139.

[41] D. S. Grebenkov, Probability distribution of the boundary local time of reflected Brownian motion in Euclidean domains, Phys. Rev. E 100 (6) (2019) 062110. 
[42] T. R. Ginn, On the distribution of multicomponent mixtures over generalized exposure time in subsurface flow and reactive transport: Foundations, and formulations for groundwater age, chemical heterogeneity, and biodegradation, Water Resour. Res. 35 (5) (1999) 1395-1407.

[43] T. R. Ginn, On the distribution of multicomponent mixtures over generalized exposure time in subsurface flow and reactive transport: Theory and formulations for residence-time-dependent sorption/desorption with memory, Water Resour. Res. 36 (10) (2000) 2885-2893.

[44] T. R. Ginn, On the distribution of multicomponent mixtures over generalized exposure time in subsurface flow and reactive transport: Batch and column applications involving residence-time distributions and nonMarkovian reaction kinetics, Water Resour. Res. 36 (10) (2000) 28952903.

[45] U. Seeboonruang, T. R. Ginn, Upscaling heterogeneity in aquifer reactivity via exposure-time concept: Forward model, J. Contam. Hydrol. $84(3-4)(2006)$ 127-154.

[46] A. Sanz-Prat, C. Lu, R. T. Amos, M. Finkel, D. W. Blowes, O. A. Cirpka, Exposure-time based modeling of nonlinear reactive transport in porous media subject to physical and geochemical heterogeneity, J. Contam. Hydrol. 192 (2016) 35-49.

[47] T. Aquino, M. Dentz, Kinetics of contact processes under segregation, Phys. Rev. E 101 (1) (2020) 012114.

[48] P. Grassberger, I. Procaccia, The long time properties of diffusion in a medium with static traps, J. Chem. Phys. 77 (12) (1982) 6281-6284.

[49] R. Kayser, J. Hubbard, Diffusion in a medium with a random distribution of static traps, Phys. Rev. Lett. 51 (2) (1983) 79.

[50] J. Klafter, A. Blumen, G. Zumofen, Fractal behavior in trapping and reaction: A random walk study, J. Stat. Phys. 36 (5-6) (1984) 561-577.

[51] G. H. Weiss, Overview of theoretical models for reaction rates, J. of Stat. Phys. 42 (1-2) (1986) 3-36. 
[52] J.-P. Bouchaud, A. Georges, Anomalous diffusion in disordered media: statistical mechanisms, models and physical applications, Phys. Rep. 195 (4-5) (1990) 127-293.

[53] S. Yuste, L. Acedo, Some exact results for the trapping of subdiffusive particles in one dimension, Physica A Stat. Mech. Appl. 336 (3-4) (2004) $334-346$.

[54] G. J. Lapeyre, M. Dentz, Reaction-diffusion with stochastic decay rates, Phys. Chem. Chem. Phys. 19 (2017) 18863-18879.

[55] T. Aquino, G. J. Lapeyre, M. Dentz, Survival and confinement under quenched disorder, Physical Chemistry Chemical Physics 21 (42) (2019) 23598-23610.

[56] F. Golfier, C. Zarcone, B. Bazin, R. Lenormand, D. Lasseux, M. Quintard, On the ability of a darcy-scale model to capture wormhole formation during the dissolution of a porous medium, J. Fluid Mech. 457 (2002) 213.

[57] Y. Edery, H. Scher, B. Berkowitz, Dissolution and precipitation dynamics during dedolomitization, Water Resour. Res. 47 (8) (2011).

[58] C. Garing, P. Gouze, M. Kassab, M. Riva, A. Guadagnini, Anticorrelated porosity permeability changes during the dissolution of carbonate rocks: experimental evidences and modeling, Transp. Porous Media 107 (2) (2015) 595-621.

[59] R. Liyanage, J. Cen, S. Krevor, J. P. Crawshaw, R. Pini, Multidimensional observations of dissolution-driven convection in simple porous media using x-ray ct scanning, Transp. Porous Media 126 (2) (2019) 355378 .

[60] H. P. Menke, B. Bijeljic, M. G. Andrew, M. J. Blunt, Dynamic threedimensional pore-scale imaging of reaction in a carbonate at reservoir conditions, Environ. Sci. Tech. 49 (7) (2015) 4407-4414.

[61] B. Noetinger, D. Roubinet, A. Russian, T. Le Borgne, F. Delay, M. Dentz, J.-R. De Dreuzy, P. Gouze, Random walk methods for modeling hydrodynamic transport in porous and fractured media from pore to reservoir scale, Transp. Porous Media 115 (2) (2016) 345-385. 
[62] N. Sund, T. Aquino, D. Bolster, Effective models for transport in complex heterogeneous hydrologic systems, in: P. Maurice (Ed.), Encyclopedia of Water: Science, Technology, and Society, John Wiley \& Sons, 2019 .

[63] N. G. Van Kampen, Stochastic processes in physics and chemistry, Vol. 1, Elsevier, 1992.

[64] G. Sole-Mari, D. Fernàndez-Garcia, P. Rodríguez-Escales, X. SanchezVila, A kde-based random walk method for modeling reactive transport with complex kinetics in porous media, Water Resour. Res. 53 (11) (2017) 9019-9039.

[65] G. Sole-Mari, D. Bolster, D. Fernàndez-Garcia, X. Sanchez-Vila, Particle density estimation with grid-projected and boundary-corrected adaptive kernels, Adv. Water Resour. 131 (2019) 103382.

[66] S. Whitaker, The method of volume averaging, Vol. 13, Springer Science \& Business Media, 2013.

[67] D. Bolster, A. Paster, D. A. Benson, A particle number conserving Lagrangian method for mixing-driven reactive transport, Water Resour. Res. 52 (2) (2016) 1518-1527.

[68] K. Develi, T. Babadagli, Quantification of natural fracture surfaces using fractal geometry, Math. Geol. 30 (8) (1998) 971-998.

[69] W. Feller, An introduction to probability theory and its applications, Vol. 2, John Wiley \& Sons, 2008.

[70] T. Aquino, A. Aubeneau, G. McGrath, D. Bolster, S. Rao, Noise-driven return statistics: Scaling and truncation in stochastic storage processes, Sci. Rep. 7 (1) (2017) 1-6.

[71] T. Aquino, M. Dentz, A coupled time domain random walk approach for transport in media characterized by broadly-distributed heterogeneity length scales, Adv. Water Resour. 119 (2018) 60-69.

[72] D. A. Benson, R. Schumer, M. M. Meerschaert, Recurrence of extreme events with power-law interarrival times, Geophys. Res. Lett. 34 (16) (2007). 
[73] D. A. Benson, M. M. Meerschaert, A simple and efficient random walk solution of multi-rate mobile/immobile mass transport equations, Adv. Water Resour. 32 (4) (2009) 532-539.

[74] M. M. Meerschaert, A. Sikorskii, Stochastic models for fractional calculus, Vol. 43, Walter de Gruyter, 2012.

[75] R. Turuban, D. R. Lester, J. Heyman, T. Le Borgne, Y. Méheust, Chaotic mixing in crystalline granular media, J. Fluid Mech. 871 (2019) $562-594$.

[76] S. H. Simon, The Oxford solid state basics, OUP Oxford, 2013.

[77] D. R. Lester, M. Dentz, T. Le Borgne, Chaotic mixing in threedimensional porous media, J. Fluid Mech. 803 (2016) 144-174.

[78] J. Heyman, D. R. Lester, R. Turúban, Y. Méheust, T. Le Borgne, Stretching and folding sustain microscale chemical gradients in porous media, Proc. Natl. Acad. Sci. U.S.A. 117 (24) (2020) 13359-13365.

[79] J. Heyman, D. Lester, T. Le Borgne, Scalar signatures of chaotic mixing in porous media, Physical Review Letters 126 (3) (2021) 034505.

[80] S. Condamin, O. Bénichou, V. Tejedor, R. Voituriez, J. Klafter, Firstpassage times in complex scale-invariant media, Nature 450 (7166) (2007) $77-80$.

[81] A. F. Atiya, S. A. Metwally, Efficient estimation of first passage time density function for jump-diffusion processes, SIAM J. Sci. Comput. 26 (5) (2005) 1760-1775.

[82] D. Shalloway, A. K. Faradjian, Efficient computation of the first passage time distribution of the generalized master equation by steady-state relaxation, J. Chem. Phys. 124 (5) (2006) 054112.

[83] D. Schnoerr, B. Cseke, R. Grima, G. Sanguinetti, Efficient low-order approximation of first-passage time distributions, Phys. Rev. Lett. 119 (21) (2017) 210601. 
[84] D. A. Benson, T. Aquino, D. Bolster, N. Engdahl, C. V. Henri, D. Fernandez-Garcia, A comparison of Eulerian and Lagrangian transport and non-linear reaction algorithms, Adv. Water Resour. 99 (2017) $15-37$.

[85] T. Aquino, T. Le Borgne, The diffusing-velocity random walk: a spatialMarkov formulation of heterogeneous advection and diffusion, J. Fluid Mech. 910 (2021) A12.

[86] The CGAL Project, CGAL User and Reference Manual, 5.1.1 Edition, CGAL Editorial Board, 2020.

URL https://doc.cgal.org/5.1.1/Manual/packages.html

[87] The OpenFOAM Foundation, OpenFOAM v8 User Guide (2020).

URL https://cfd.direct/openfoam/user-guide 
1457 Declaration of Competing Interest

1458 The authors declare that they have no known competing financial inter1459 ests or personal relationships that could have appeared to influence the work 1460 reported in this paper. 
1461

${ }_{1462}$ Tomás Aquino: Conceptualization, Methodology, Software, Writing 1463 original draft.

${ }_{1464}$ Tanguy Le Borgne: Conceptualization, Writing - review \& editing. 